\title{
Emission of Massive Scalar Fields by a Higher-Dimensional Rotating Black-Hole
}

\author{
P. Kanti and N. Pappas \\ Division of Theoretical Physics, Department of Physics, \\ University of Ioannina, Ioannina GR-45110, Greece
}

\begin{abstract}
We perform a comprehensive study of the emission of massive scalar fields by a higherdimensional, simply rotating black hole both in the bulk and on the brane. We derive approximate, analytic results as well as exact numerical ones for the absorption probability, and demonstrate that the two sets agree very well in the low and intermediate-energy regime for scalar fields with mass $m_{\Phi} \leq 1 \mathrm{TeV}$ in the bulk and $m_{\Phi} \leq 0.5 \mathrm{TeV}$ on the brane. The numerical values of the absorption probability are then used to derive the Hawking radiation power emission spectra in terms of the number of extra dimensions, angular-momentum of the black hole and mass of the emitted field. We compute the total emissivities in the bulk and on the brane, and demonstrate that, although the brane channel remains the dominant one, the bulk-over-brane energy ratio is considerably increased (up to 33\%) when the mass of the emitted field is taken into account.
\end{abstract}




\section{Introduction}

The postulation of the existence of additional spacelike dimensions in nature, that can be as large as a few micrometers [1] or even infinite in size [2], has led to the idea of a higher-dimensional gravitational theory with a fundamental energy scale $M_{*}$ much smaller than the traditional Planck scale $M_{P}$. If this can be realised with $M_{*}$ close to the $\mathrm{TeV}$ scale, present of future experiments may accelerate particles at energies beyond this new gravity scale. This will unavoidably lead to the occurence of strong gravity effects in particle collisions and the production of heavy final states, including miniature black holes [3].

The lifetime of these black holes is expected to be very short as they instantaneously decay via the emission of Hawking radiation [4] (for detailed reviews of their properties, see [5, 6]). Since these black holes will be created and decay in front of our detectors, it is anticipated that the emission of Hawking radiation will be the main obervable signature of their creation and, at the same time, a manifestation of the existence of additional spacelike dimensions in nature. As a result, the study of the emission of radiation by higher-dimensional black holes has been the subject of an intensive research activity over the last years. This includes the emission from both spherically-symmetric [7, 8, 19, 10, 11, 12, 13, 14, 15, 16] and rotating [17, 18, 19, 20, 21, 22, 23, 24, 25, 26, 27, 28, 29, 30, 31, 32] black holes in the form of zero and non-zero spin fields.

In order to simplify the analysis, the emitted fields are assumed to be minimallycoupled to gravity but otherwise free as well as massless. Nevertheless, in the context of the four-dimensional analysis [33] it was found that for certain particles and mass of the black hole, the particle mass can significantly (up to 50\%) suppress the emission rate. Recently, a set of works [34] has addressed the question of the role of the mass of the emitted field (as well as that of the charge) for emission on the brane by a higher-dimensional black hole. Here, we extend this analysis by considering the case of a higher-dimensional black hole with a non-vanishing angular momentum emitting massive scalar fields. We perform a comprehensive study of the absorption probability and energy emission rate for a range of values of the mass of the emitted field, number of extra dimensions, and angular momentum of the black hole. By integrating over the entire frequency range, we compute the total emissivities and obtain the suppression factors in each case. We also consider the cases of both bulk and brane emission, and pose the additional question

of whether the presence of the mass of the emitted field can affect the bulk-over-brane energy ratio and threaten the dominance of the brane channel.

The outline of this paper is as follows: In section 2, we study the emission of massive scalar fields by a higher-dimensional, simply rotating black hole in the bulk; we compute the value of the absorption probability both analytically and numerically and compare the two sets of results; finally, we derive the exact energy emission spectra and discuss their behaviour. In section 3, we turn to the brane and perform the same tasks. The total emissivities for bulk and brane emission are derived in section 4, and the bulk-over-brane ratio is computed for a large number of values of the parameters of the theory. We close with our conclusions in section 5 . 


\section{Emission of Massive Scalars in the Bulk}

In this work, we will consider the case of a higher-dimensional, neutral, simply rotating black hole whose gravitational background is described by the following form of the Myers-Perry solution [35]

$$
\begin{aligned}
d s^{2}=-(1 & \left.-\frac{\mu}{\Sigma r^{n-1}}\right) d t^{2}-\frac{2 a \mu \sin ^{2} \theta}{\Sigma r^{n-1}} d t d \varphi+\frac{\Sigma}{\Delta} d r^{2}+\Sigma d \theta^{2} \\
& +\left(r^{2}+a^{2}+\frac{a^{2} \mu \sin ^{2} \theta}{\Sigma r^{n-1}}\right) \sin ^{2} \theta d \varphi^{2}+r^{2} \cos ^{2} \theta d \Omega_{n}^{2},
\end{aligned}
$$

where

$$
\Delta=r^{2}+a^{2}-\frac{\mu}{r^{n-1}}, \quad \Sigma=r^{2}+a^{2} \cos ^{2} \theta,
$$

and $d \Omega_{n}^{2}\left(\theta_{1}, \theta_{2}, \ldots, \theta_{n-1}, \phi\right)$ is the line-element on a unit $n$-sphere. The above line-element is expected to describe black holes created by an on-brane collision of particles that acquire only one non-zero angular momentum component, parallel to our brane. The black hole's mass $M_{B H}$ and angular momentum $J$ are then related to the parameters $\mu$ and $a$, respectively, as follows

$$
M_{B H}=\frac{(n+2) A_{n+2}}{16 \pi G_{D}} \mu, \quad J=\frac{2}{n+2} M_{B H} a,
$$

with $G_{D}$ being the $(4+n)$-dimensional Newton's constant, and $A_{n+2}$ the area of a $(n+2)$ dimensional unit sphere given by $A_{n+2}=2 \pi^{(n+3) / 2} / \Gamma[(n+3) / 2]$. The black hole's horizon radius $r_{h}$ follows from the equation $\Delta\left(r_{h}\right)=0$, and may be written as $r_{h}^{n+1}=\mu /\left(1+a_{*}^{2}\right)$, where $a_{*}=a / r_{h}$.

A massive scalar field, with mass $m_{\Phi}$, propagating in the gravitational background (1) will satisfy the equation of motion

$$
\frac{1}{\sqrt{-G}} \partial_{M}\left(\sqrt{-G} G^{M N} \partial_{N} \Phi\right)-m_{\Phi}^{2} \Phi=0
$$

where $G_{M N}$ the higher-dimensional metric tensor and $G$ its determinant satisfying the relation

$$
\sqrt{-G}=\Sigma \sin \theta r^{n} \cos ^{n} \theta \prod_{i=1}^{n-1} \sin ^{i} \theta_{i}
$$

Even in the presence of the mass term, the above equation can be separated [17, 36] by assuming the factorised ansatz

$$
\Phi=e^{-i \omega t} e^{i m \varphi} R(r) S(\theta) Y_{\ln }\left(\theta_{1}, \ldots, \theta_{n-1}, \phi\right),
$$

where $Y_{l n}\left(\theta_{1}, \ldots, \theta_{n-1}, \phi\right)$ are the hyperspherical harmonics on the $n$-sphere that satisfy the equation 37,38 ]

$$
\sum_{k=1}^{n-1} \frac{1}{\prod_{i=1}^{n-1} \sin ^{i} \theta_{i}} \partial_{\theta_{k}}\left[\left(\prod_{i=1}^{n-1} \sin ^{i} \theta_{i}\right) \frac{\partial_{\theta_{k}} Y_{l n}}{\prod_{i>k}^{n-1} \sin ^{2} \theta_{i}}\right]+\frac{\partial_{\phi \phi} Y_{l n}}{\prod_{i=1}^{n-1} \sin ^{2} \theta_{i}}+l(l+n-1) Y_{l n}=0 .
$$


The functions $R(r)$ and $S(\theta)$ in turn satisfy the following decoupled radial and angular equation

$$
\begin{gathered}
\frac{1}{r^{n}} \partial_{r}\left(r^{n} \Delta \partial_{r} R\right)+\left(\frac{K^{2}}{\Delta}-\frac{l(l+n-1) a^{2}}{r^{2}}-\tilde{\Lambda}_{j \ell m}-m_{\Phi}^{2} r^{2}\right) R=0 \\
\frac{1}{\sin \theta \cos ^{n} \theta} \partial_{\theta}\left(\sin \theta \cos ^{n} \theta \partial_{\theta} S\right)+\left(\tilde{\omega}^{2} a^{2} \cos ^{2} \theta-\frac{m^{2}}{\sin ^{2} \theta}-\frac{l(l+n-1)}{\cos ^{2} \theta}+\tilde{E}_{j \ell m}\right) S=0,
\end{gathered}
$$

respectively. In the above,

$$
K=\left(r^{2}+a^{2}\right) \omega-a m, \quad \tilde{\Lambda}_{j \ell m}=\tilde{E}_{j \ell m}+a^{2} \omega^{2}-2 a m \omega .
$$

For the above decoupling to take place, the angular function $S(\theta)$ needs to satisfy a modified higher-dimensional spheroidal harmonics equation: compared to the massless case [39], it has the energy $\omega$ replaced by the momentum $\tilde{\omega} \equiv \sqrt{\omega^{2}-m_{\Phi}^{2}}$. Then, the massive angular eigenvalue $\tilde{E}_{j \ell m}(a \tilde{\omega})$ is related to the massless one $E_{j \ell m}(a \omega)$ by merely a shift of its argument: $a \omega \rightarrow a \tilde{\omega}$. Here, we will employ the power-series expression of the angular eigenvalue [39] in terms of the parameter $(a \omega)$ which, under the aforementioned shift and up to 5 th order, takes the form

$$
\begin{aligned}
\tilde{E}_{j l m} & =j(j+n+1)-(a \tilde{\omega})^{2} \frac{\left[-1+2 l(l-1)+2 j(j+1)-2 m^{2}+2 n(j+l)+n^{2}\right]}{(2 j+n-1)(2 j+n+3)} \\
& +(a \tilde{\omega})^{4}\left\{\frac{(l-j+|m|)(l+j-|m|+n-1)}{16(2 j+n-3)(2 j+n-1)^{2}}[(2+l-j+|m|)(l+j-|m|+n-3)\right. \\
& \left.-4(2 j+n-3) \frac{\left[-1+2 l(l-1)+2 j(j+1)-2 m^{2}+2 n(j+l)+n^{2}\right]}{(2 j+n-1)(2 j+n+3)}\right] \\
& -\frac{(l-j+|m|-2)(l+j+n-|m|+1)}{16(2 j+5+n)(2 j+n+3)^{2}}[(l-j+|m|-4)(j+l+n-|m|+3) \\
& \left.\left.+4(2 j+n+5) \frac{\left[-1+2 l(l-1)+2 j(j+1)-2 m^{2}+2 n(j+l)+n^{2}\right]}{(2 j+n-1)(2 j+n+3)}\right]\right\} \\
& +\mathcal{O}\left((a \tilde{\omega})^{6}\right) .
\end{aligned}
$$

The analytic form of the angular eigenvalue was studied in detail in the context of previous works focusing on the emission of massless scalars [29, 30] and gravitons [31] in the bulk. It was found that its value, when terms up to 5th order or higher are kept, is remarkably close to the exact numerical value and that considerable deviations appear only for a very large angular momentum of the black hole or energy of the emitted particle, that lie beyond the range of values considered in this work. For this reason, the analytic form (11) of the angular eigenvalue will be employed in the derivation of the absorption probability in both an analytic and numerical method. We should still demand of course the convergence of the power series by imposing restrictions on the allowed values of the integer parameters $(j, \ell, m)$ that specify the emission mode: $m$, that denotes the angular momentum of the mode along our brane, may take any integer value while $\ell$ and $j$ - the angular momentum number in the $n$-sphere and total angular momentum number, respectively - may take any positive or zero integer value provided [39] that $j \geq \ell+|m|$ and $\frac{j-(\ell+|m|)}{2} \in\left\{0, \mathbb{Z}^{+}\right\}$. 


\subsection{The Absorption Probability in the Bulk}

For the derivation of the absorption probability $\left|\mathcal{A}_{j \ell m}\right|^{2}$ we need the solution for the radial function $R(r)$. We will first solve Eq. (8) analytically by using an approximate method, and we will derive an analytic expression for the absorption probability which in principle is valid in the low-energy and low-angular-momentum limit. We will then solve the same equation numerically to derive the exact value of $\left|\mathcal{A}_{j \ell m}\right|^{2}$, that will subsequently be used to derive the Hawking radiation spectrum. The two sets of results will be compared, and the validity of the approximate method will be studied in terms of the value of the angular-momentum parameter $a$, number of extra dimensions $n$ and mass of the emitted particle $m_{\Phi}$.

The approximate analytic method amounts to solving the radial equation in the two asymptotic regimes, those of the black-hole horizon and far away from it, and then matching them in an intermediate regime. Apart from the appearance of the mass parameter $m_{\Phi}$, the analysis is very similar to the one for the emission of massless scalar fields in the bulk which has already appeared in the literature [29]. Therefore, here we briefly present the analysis and results giving emphasis to the differences arising due to the presence of the mass term.

In terms of the new radial variable $r \rightarrow f(r)=\Delta(r) /\left(r^{2}+a^{2}\right)$ [27, 28], the radial equation (8) near the horizon $\left(r \simeq r_{h}\right)$ takes the form

$$
f(1-f) \frac{d^{2} R}{d f^{2}}+\left(1-D_{*} f\right) \frac{d R}{d f}+\left[\frac{K_{*}^{2}}{A_{*}^{2} f(1-f)}-\frac{C_{*}}{A_{*}^{2}(1-f)}\right] R=0,
$$

where $A_{*} \equiv(n+1)+(n-1) a_{*}^{2}, D_{*} \equiv 1-4 a_{*}^{2} / A_{*}^{2}$, while $K_{*}$ and $C_{*}$ are defined as

$$
K_{*}=\left(1+a_{*}^{2}\right) \omega r_{h}-a_{*} m, \quad C_{*}=\left[\ell(\ell+n-1) a_{*}^{2}+\tilde{\Lambda}_{j \ell m}+m_{\Phi}^{2} r_{h}^{2}\right]\left(1+a_{*}^{2}\right),
$$

respectively. By employing the transformation $R_{N H}(f)=f^{\alpha}(1-f)^{\beta} F(f)$ the above equation takes the form of a hypergeometric differential equation [40] as long as

$$
\alpha_{ \pm}= \pm \frac{i K_{*}}{A_{*}}, \quad \beta=\frac{1}{2}\left[\left(2-D_{*}\right)-\sqrt{\left(D_{*}-2\right)^{2}-4\left(\frac{K_{*}^{2}-C_{*}}{A_{*}^{2}}\right)}\right] .
$$

The radial function $R_{N H}(f)$ must satisfy the boundary condition that no outgoing modes exist near the black-hole horizon which then reduces the general solution of the hypergeometric equation to the physically acceptable one

$$
R_{N H}(f)=A_{-} f^{\alpha}(1-f)^{\beta} F(a, b, c ; f),
$$

with $a=\alpha+\beta+D_{*}-1, b=\alpha+\beta, c=1+2 \alpha$ and $A_{-}$an integration constant. Indeed, we may easily check that in the limit $r \rightarrow r_{h}$ (or equivalently $f \rightarrow 0$ ), and by making the choice $\alpha=\alpha_{-}$, we obtain

$$
R_{N H}(f) \simeq A_{-} f^{-i K_{*} / A_{*}}=A_{-} e^{-i k y}
$$


that has a form of an incoming plane-wave, as expected, in terms of a tortoise-like coordinate defined by $y=r_{h}\left(1+a_{*}^{2}\right) \ln (f) / A_{*}$. In the above, $k$ is given by

$$
k \equiv \frac{K_{*}}{r_{h}\left(1+a_{*}^{2}\right)}=\omega-m \Omega_{h}=\omega-\frac{m a}{r_{h}^{2}+a^{2}},
$$

where $\Omega_{h}$ is the rotation velocity of the black hole.

In the far-field regime $\left(r \gg r_{h}\right)$, the substitution $R(r)=r^{-\left(\frac{n+1}{2}\right)} \tilde{R}(r)$ brings Eq. (8) into the form of a Bessel equation [40]

$$
\frac{d^{2} \tilde{R}}{d z^{2}}+\frac{1}{z} \frac{d \tilde{R}}{d z}+\left(1-\frac{\tilde{E}_{j \ell m}+a^{2} \tilde{\omega}^{2}+\left(\frac{n+1}{2}\right)^{2}}{z^{2}}\right) \tilde{R}=0
$$

in terms of $z \equiv \tilde{\omega} r$, with solution

$$
R_{F F}(r)=\frac{B_{1}}{r^{\frac{n+1}{2}}} J_{\nu}(\tilde{\omega} r)+\frac{B_{2}}{r^{\frac{n+1}{2}}} Y_{\nu}(\tilde{\omega} r) .
$$

In the above, $J_{\nu}$ and $Y_{\nu}$ are the Bessel functions of the first and second kind, respectively, and $\nu=\sqrt{\tilde{E}_{j \ell m}+a^{2} \tilde{\omega}^{2}+\left(\frac{n+1}{2}\right)^{2}}$.

We now need to smoothly match the two asymptotic solutions (15) and (19) in an intermediate regime. The near-horizon solution (15) must first be shifted, so that its argument changes from $f$ to $(1-f)$, and subsequently expanded in the $r \gg r_{h}$ limit. Then, it takes the polynomial form

$$
R_{N H}(r) \simeq A_{1} r^{-(n+1) \beta}+A_{2} r^{(n+1)\left(\beta+D_{*}-2\right)},
$$

with $A_{1}$ and $A_{2}$ defined as

$$
\begin{aligned}
& A_{1}=A_{-}\left[\left(1+a_{*}^{2}\right) r_{h}^{n+1}\right]^{\beta} \frac{\Gamma(c) \Gamma(c-a-b)}{\Gamma(c-a) \Gamma(c-b)} \\
& A_{2}=A_{-}\left[\left(1+a_{*}^{2}\right) r_{h}^{n+1}\right]^{-\left(\beta+D_{*}-2\right)} \frac{\Gamma(c) \Gamma(a+b-c)}{\Gamma(a) \Gamma(b)} .
\end{aligned}
$$

The far-field solution (19) is in turn expanded to small values of $r$ leading to

$$
R_{F F}(r) \simeq \frac{B_{1}\left(\frac{\tilde{\omega} r}{2}\right)^{\nu}}{r^{\frac{n+1}{2}} \Gamma(\nu+1)}-\frac{B_{2}}{\pi r^{\frac{n+1}{2}}} \frac{\Gamma(\nu)}{\left(\frac{\tilde{\omega} r}{2}\right)^{\nu}} .
$$

The two polynomial forms match perfectly if we take the small $a_{*}$ and $\tilde{\omega}_{*}$ limit in the power coefficients of $r$. In that case we can ignore terms of order $\left(\tilde{\omega}_{*}^{2}, a_{*}^{2}, a_{*} \tilde{\omega}_{*}\right)$ or higher, and obtain $-(n+1) \beta \simeq j,(n+1)\left(\beta+D_{*}-2\right) \simeq-(j+n+1)$, and $\nu \simeq j+(n+1) / 2$. We then demand the matching of the corresponding multiplicative coefficients, which leads to a constraint for the far-asymptotic integration constants $B_{1}$ and $B_{2}$, namely

$$
\begin{aligned}
B \equiv & \frac{B_{1}}{B_{2}}=-\frac{1}{\pi}\left(\frac{2}{\tilde{\omega} r_{h}\left(1+a_{*}^{2}\right)^{\frac{1}{n+1}}}\right)^{2 j+n+1} \sqrt{\tilde{E}_{j \ell m}+a^{2} \tilde{\omega}^{2}+\left(\frac{n+1}{2}\right)^{2}} \\
& \times \frac{\Gamma^{2}\left(\sqrt{\tilde{E}_{j \ell m}+a^{2} \tilde{\omega}^{2}+\left(\frac{n+1}{2}\right)^{2}}\right) \Gamma\left(\alpha+\beta+D_{*}-1\right) \Gamma(\alpha+\beta) \Gamma\left(2-2 \beta-D_{*}\right)}{\Gamma\left(2 \beta+D_{*}-2\right) \Gamma\left(2+\alpha-\beta-D_{*}\right) \Gamma(1+\alpha-\beta)},
\end{aligned}
$$


that guarantees the existence of a smooth, analytic solution for the radial part of the wavefunction for all $r$, valid in the low-energy and low-rotation limit. We stress that, in order to achieve a higher level of accuracy in our analysis, no expansion is performed in the arguments of the Gamma functions. This method has been used in the literature before to derive analytic solutions for brane [27] and bulk [29] massless scalar fields. In both cases, the analytic results were shown to be in excellent agreement with the exact numerical ones in the low-energy regime and quite often at the intermediate-energy regime too.

In the presence of the mass term, though, there is one more constraint that needs to be satisfied for the perfect match to take place. In the low-energy and low-angularmomentum limit, the expression for the parameter $\beta$, Eq. (14), becomes

$$
\beta \simeq \frac{1}{2}\left[1-\frac{1}{(n+1)} \sqrt{(2 j+n+1)^{2}+4 m_{\Phi}^{2} r_{h}^{2}}\right] .
$$

For $j \geq 0$ and $n \geq 1$, we thus need to satisfy $m_{\Phi} r_{h}<1$. In order to derive some quantitative results, let us assume that $M_{*}=1 \mathrm{TeV}$ and $M_{B H}=5 \mathrm{TeV}$. If we ignore for a moment the angular momentum of the black hole and use the mass-horizon radius relation for a higher-dimensional Schwarzschild black hole, we find $r_{h} \simeq(4-2) 10^{-4} \mathrm{fm}$ for $n=1-7$, respectively [5]. Then, the aforementioned constraint on the mass of the bulk scalar field translates to

$$
m_{\Phi}<(0.5-1) \mathrm{TeV}, \quad \text { for } \quad n=1-7 .
$$

If we reinstate the angular momentum of the black hole, then the value of the black-hole horizon, for the same mass, becomes smaller since $r_{h}^{n+1}=\mu /\left(1+a_{*}^{2}\right)$; therefore, the upper bound on the mass of the scalar field increases further and becomes easier to satisfy.

In order to define the absorption probability, we finally expand the far-field solution (19) for $r \rightarrow \infty$, and obtain

$$
\begin{aligned}
R_{F F}(r) & \simeq \frac{1}{r^{\frac{n+2}{2}} \sqrt{2 \pi \tilde{\omega}}}\left[\left(B_{1}+i B_{2}\right) e^{-i\left(\tilde{\omega} r-\frac{\pi}{2} \nu-\frac{\pi}{4}\right)}+\left(B_{1}-i B_{2}\right) e^{i\left(\tilde{\omega} r-\frac{\pi}{2} \nu-\frac{\pi}{4}\right)}\right] \\
& =A_{\text {in }}^{(\infty)} \frac{e^{-i \tilde{\omega} r}}{r^{\frac{n+2}{2}}}+A_{\text {out }}^{(\infty)} \frac{e^{i \tilde{\omega} r}}{r^{\frac{n+2}{2}}}
\end{aligned}
$$

which readily leads to

$$
\left|\mathcal{A}_{j \ell m}\right|^{2}=1-\left|\frac{A_{\text {out }}^{(\infty)}}{A_{\text {in }}^{(\infty)}}\right|^{2}=1-\left|\frac{B_{1}-i B_{2}}{B_{1}+i B_{2}}\right|^{2}=\frac{2 i\left(B^{*}-B\right)}{B B^{*}+i\left(B^{*}-B\right)+1} .
$$

The above expression, in conjunction with Eq. (23), is our final analytic result for the absorption probability for massive scalar fields emitted in the bulk by a higherdimensional, simply-rotating black hole. Summarizing all of the aforementioned assumptions, it is valid as long as the energy and mass of the emitted particle and the angularmomentum of the black hole stay below unity (in units of $r_{h}^{-1}$ and $r_{h}$, respectively). Its range of validity will be shortly investigated in terms of the values of the above parameters, as well as that of the number of extra dimensions $n$. 
Equation (27) is also useful for studying analytically various aspects of the absorption probability such as its behaviour in the superradiant regime and the asymptotic limit $\tilde{\omega} \rightarrow 0$. If we expand Eq. (27) in the low-energy limit, a more convenient form may be derived for both purposes - a similar analysis was presented in all detail in [27] where the emission of massless scalar fields on the brane by the same type of black hole was studied. From Eq. (23) we see that, in that limit, we obtain $B \propto 1 / \tilde{\omega}^{2 j+n+1}$, and therefore

$$
\left|\mathcal{A}_{j \ell m}\right|^{2} \simeq 2 i\left(\frac{1}{B}-\frac{1}{B^{*}}\right)=\Sigma_{1} \times \Sigma_{2} \times \Sigma_{3}
$$

where

$$
\begin{gathered}
\Sigma_{1}=\frac{-2 i \pi\left(\tilde{\omega} r_{h} / 2\right)^{2 j+n+1}}{\left(j+\frac{n+1}{2}\right) \Gamma^{2}\left(j+\frac{n+1}{2}\right)} \frac{\left(1+a_{*}^{2}\right)^{\frac{2 j+n+1}{n+1}} \Gamma\left(2 \beta+D_{*}-2\right)}{\Gamma\left(2-2 \beta-D_{*}\right)}, \\
\Sigma_{2}=\frac{1}{\left|\Gamma\left(\alpha+\beta+D_{*}-1\right)\right|^{2}|\Gamma(\alpha+\beta)|^{2}}
\end{gathered}
$$

and

$$
\begin{aligned}
\Sigma_{3} & =\Gamma\left(2+\alpha-\beta-D_{*}\right) \Gamma\left(-\alpha+\beta+D_{*}-1\right) \Gamma(1+\alpha-\beta) \Gamma(-\alpha+\beta)-(c c) \\
& =\frac{-\pi^{2} \sin (2 \pi \alpha) \sin \pi\left(2 \beta+D_{*}\right)}{\sin \pi\left(\alpha+\beta+D_{*}\right) \sin \pi\left(-\alpha+\beta+D_{*}\right) \sin \pi(\alpha+\beta) \sin \pi(-\alpha+\beta)} .
\end{aligned}
$$

The $(c c)$ term above stands for the complex conjugate of the corresponding expression. As the energy of the emitted mode decreases, moving towards the asymptotic limit $\tilde{\omega} \rightarrow 0$, for modes with $m>0$, we meet the value $\omega=\omega_{s} \equiv m \Omega_{h}$. From Eqs. (14) and (17), it is clear that for that value $\alpha \rightarrow 0$, in which case Eq. (28) gets simplified to

$$
\left|\mathcal{A}_{j \ell m}\right|^{2}=\frac{4 \pi\left(\tilde{\omega} r_{h} / 2\right)^{2 j+n+1} K_{*} \sin ^{2} \pi\left(2 \beta+D_{*}\right) \Gamma^{2}\left(2 \beta+D_{*}-2\right) \Gamma^{2}(1-\beta)\left(2-D_{*}-2 \beta\right)}{A_{*}\left(1+a_{*}^{2}\right)^{-\frac{2 j+n+1}{n+1}}\left(j+\frac{n+1}{2}\right) \Gamma^{2}\left(j+\frac{n+1}{2}\right) \Gamma^{2}\left(\beta+D_{*}-1\right) \sin ^{2} \pi\left(\beta+D_{*}\right)} .
$$

In the above expression, all terms are positive definite, including the $\left(2-D_{*}-2 \beta\right)$ one, apart from $K_{*}$ whose sign, as expected, defines the sign of the absorption probability: for $\omega<\omega_{s},\left(\omega-m \Omega_{h}\right)$ takes a negative value signalling the occurence of superradiance.

For modes with $m \leq 0$, there is no superradiance effect, and we may thus approach the asymptotic limit $\tilde{\omega} \rightarrow 0$. From the coefficient $\left(\tilde{\omega} r_{h}\right)^{2 j+n+1}$ in the expression of $\Sigma_{1}$ it is clear that, in the massive case, too, it is the lowest partial modes that dominate the value of the absorption probability in the low-energy regime. We will therefore focus our attention on the dominant mode $j=\ell=m=0$, and derive the behaviour of the absorption probability in the above asymptotic limit. Although for massive modes with $m \leq 0$ the parameter $\alpha$ never becomes exactly zero, it acquires its smallest possible value as $\tilde{\omega} \rightarrow 0$. Equation (32) therefore remains approximately valid, and, for $j=\ell=m=0$ and $\beta=0+\mathcal{O}\left(\tilde{\omega}^{2}\right)$, it is simplified further to give

$$
\left|\mathcal{A}_{000}\right|^{2}=\frac{4 \pi\left(1+a_{*}^{2}\right)^{2}\left(\tilde{\omega} r_{h}\right)^{n+1} \omega r_{h}}{A_{*} 2^{n}(n+1) \Gamma^{2}\left(\frac{n+1}{2}\right)\left(2-D_{*}\right)}+\ldots .
$$


We may also compute the absorption cross-section $\sigma_{000}$ for the dominant massive scalar bulk mode in the asymptotic low-energy regime by using the formula [41, 29]

$$
\sigma_{j \ell m}(\omega)=\frac{2^{n}}{\pi} \Gamma^{2}\left(\frac{n+3}{2}\right) \frac{A_{H}}{\left(\tilde{\omega} r_{h}\right)^{n+2}} \frac{N_{\ell}}{\left(1+a_{*}^{2}\right)}\left|\mathcal{A}_{j \ell m}\right|^{2}
$$

that relates the absorption cross-section with the absorption probability for a scalar mode propagating in the background of a higher-dimensional, simply rotating black hole. In the above

$$
N_{\ell}=\frac{(2 \ell+n-1)(\ell+n-2) !}{\ell !(n-1) !}, \quad A_{H}=\frac{2 \pi^{\frac{n+3}{2}} r_{h}^{n}\left(r_{h}^{2}+a^{2}\right)}{\Gamma\left(\frac{n+3}{2}\right)}
$$

are the multiplicity of the $\ell$-th partial wave in the expansion of the wave function over the hyperspherical harmonics on the $n$-sphere [29], and the horizon area of the $(4+n)$ dimensional rotating black hole, respectively. Substituting for the absorption coefficient, we obtain

$$
\sigma_{000}(\omega) \simeq \frac{(n+1)\left(1+a_{*}^{2}\right) A_{H}}{A_{*}\left(2-D_{*}\right)}\left(\frac{\omega}{\tilde{\omega}}\right)+\ldots
$$

For $a_{*}=0$ and $m_{\Phi}=0$, the above reduces to the horizon area $A_{H}$ of a higher-dimensional, spherically-symmetric black hole, as was found in [8]. For $a_{*} \neq 0$ and $m_{\Phi}=0$, it was shown in [29, 23] that the value of $\sigma_{000}$ remains very close to the area of the corresponding rotating black hole as long as $a_{*}$ is not large. For $m_{\Phi} \neq 0$, we observe significant deviations from this behaviour as the value of the absorption cross-section for the lowest partial mode is not only energy-dependent but deviates as $\tilde{\omega} \rightarrow 0$ - this is in accordance with previous results derived in the cases of a massive field propagating in the background of a 4-dimensional Kerr [33] or of a $(4+n)$-dimensional, spherically-symmetric black hole [23. This behaviour is observed only in the case of the lowest mode; higher modes have a $\tilde{\omega}^{2 j+n+1}$ leading factor in their absorption probability, and a $\tilde{\omega}^{2 j-1}$ dependence for their absorption cross-section - for $j \geq 1$, this leads to a vanishing value in the asymptotic limit $\tilde{\omega} \rightarrow 0$.

For the derivation of the value of the absorption probability, that would be valid for arbitrary values of the energy of the emitted particle and angular momentum of the black hole, we need to solve Eq. (8) numerically. To this end, a MATHEMATICA code was constructed that numerically solved for the value of the radial function $R(r)$ from the horizon outwards. The boundary conditions for the second order differential equation was the value of $R(r)$ and its first derivative at the horizon. The asymptotic solution (16) was used for that purpose, with the boundary conditions at $r \rightarrow r_{h}$ having the form

$$
R=1, \quad \frac{d R}{d r}=-i k \frac{d y}{d r}=-\frac{i k\left(1+a_{*}^{2}\right)}{\Delta(r)} .
$$

The first condition was imposed to ensure that $\left|A_{-}\right|^{2}=1$ since no outgoing mode is allowed to exist at the horizon. The second follows readily from the asymptotic solution (16) and the use of the first condition. The integration proceeds until we reach radial infinity (in practice, this happens for $r \simeq 1000 r_{h}$ ) where, according to Eq. (26)), the radial function is a superposition of incoming and outgoing modes. The corresponding 

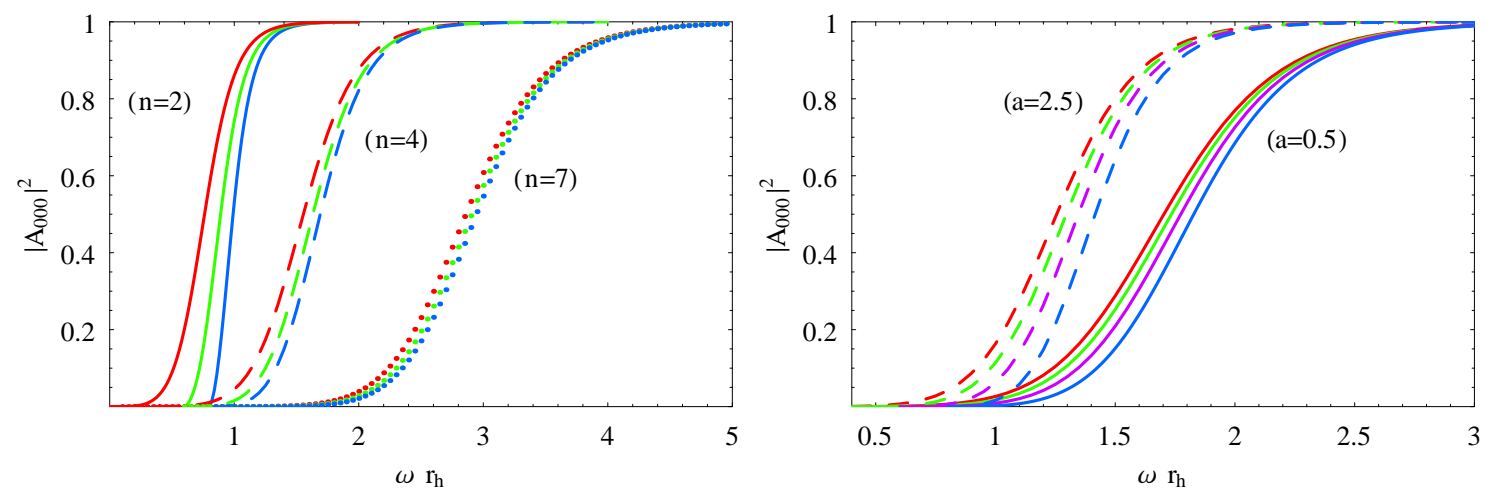

Figure 1: Absorption probabilities for the bulk scalar mode $j=\ell=m=0$, for (a) $a_{*}=$ $1, m_{\Phi}=0,0.6,0.8$ (from left to right) and various $n$, and (b) $n=4, m_{\Phi}=0,0.4,0.6,0.8$ (from left to right) and various $a_{*}$.

amplitudes are then isolated and the value of the absorption coefficient follows by use of the definition (27).

As a consistency check, we have succesfully reproduced the numerical results presented in [30] for the case of massless scalar fields emitted in the bulk by a simply rotating black hole - the case with $m_{\Phi}=0$ is also included in our plots for the easy comparison with the massless case. In Fig. 1 we plot the absorption probability for the dominant mode $j=\ell=m=0$ as a function of the three parameters, $m_{\Phi}, n$ and $a$, respectively. Figure 1(a) was drawn for fixed angular-momentum parameter $\left(a_{*}=1\right)$, and depicts the dependence of $\left|\mathcal{A}_{000}\right|^{2}$ on the value of mass of the field and number of extra dimensions: we observe that as $m_{\Phi}$ increases the value of the absorption probability decreases as expected, since a larger amount of energy is necessary for the emission of an increasingly more massive field. This pattern holds independently of the value of $n$, nevertheless, the suppression with $m_{\Phi}$ becomes less important as the number of extra dimensions gets larger. Figure 1(a) reveals also that the suppression of the absorption probability with the number of extra dimensions, found previously for massless scalar fields in the bulk [29, 30], holds also for massive fields. In Fig. 1(b), we keep fixed the number of extra dimensions $(n=4)$ and vary $m_{\Phi}$ and $a_{*}$ : again the suppression with the mass of the field is evident - contrary to what happens with $n$, the suppression is more prominent as $a_{*}$ increases, particularly in the low- and intermediate-energy regimes. The enhancement of the absorption probability as $a_{*}$ itself increases, found again previously in [29, 30], persists also in the massive case.

It would be interesting to compare the exact numerical results for the value of the absorption probability with the ones following from the analytical expression (27) with $B$ given by Eq. (23). In Fig. 2 we plot both sets of results for a range of values of the parameters $m_{\Phi}, n$ and $a_{*}$ - we consider again the dominant scalar bulk mode $j=\ell=$ $m=0$. Figure 2(a) reveals that the agreement between numerical and analytical results holds for a wide range of values of the mass parameter below unity (in units of $r_{h}^{-1}$ ), as indeed expected from the discussion below Eq. (23) regarding the values of $m_{\Phi}$. On the other hand, in terms of the number of extra dimensions, the agreement is case-dependent: 

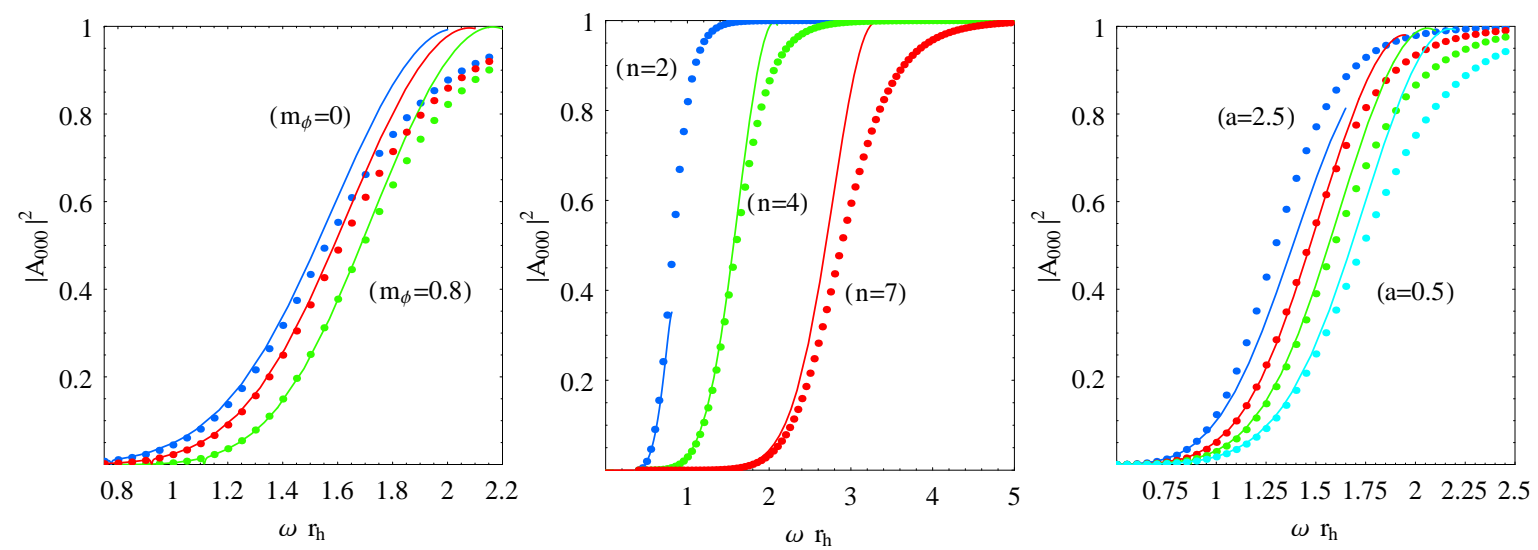

Figure 2: Comparison of the analytical (solid lines) and exact numerical (data points) results for the absorption probability for the bulk scalar mode $j=\ell=m=0$, for (a) $a_{*}=1, n=4$ and $m_{\Phi}=0,0.5,0.8$, (b) $a_{*}=1, m_{\Phi}=0.4$ and $n=2,4,7$, and (c) $n=4$, $m_{\Phi}=0.4$ and $a_{*}=0.5,1,1.5,2.5$.

as we see from Fig. 2(b), it is remarkably good for $n=4$, for $n=7$ it is limited in the lower part of the curves while for $n=2$ it stops abruptly as the analytical result suffers from the existence of poles in the arguments of the Gamma functions that force the value of $\left|\mathcal{A}_{000}\right|^{2}$ to dip towards smaller values and eventually vanish. The expression for $B$, Eq. (23), is clearly the result of an approximation method valid for small values of the angular-momentum parameter, and thus we expect the agreement between the two sets of results to become worse as the value of $a_{*}$ increases gradually; however, in Fig. 2(c), we see that the agreement is actually improving as the angular-momentum parameter increases reaching values even beyond unity, a result that holds only in the presence of the mass term of the scalar field.

Let us finally comment on the behaviour of the superradiance [42] on the parameters of the theory. In the context of the general suppression of the value of the absorption probability as the mass of the field increases, we expect that the effect of the superradiance will also be suppressed - this is indeed depicted in Fig. 3(a) where the value of $\left|\mathcal{A}_{j \ell m}\right|^{2}$ is plotted for various values of $m_{\Phi}$ for the indicative modes $(j \ell m=101)$ and $(j \ell m=202)$. Despite the observed dominance of the superradiance effect for the mode $(j \ell m=202)$ over the one for $(j \ell m=101)$, this pattern does not hold indefinitely as the angular momentum numbers increase: in fact, from Fig. 3(b), where we plot the superradiant regime for the modes $j=m=1,2, \ldots, 7$ for $m_{\Phi}=0.2, n=2$ and $a_{*}=1.5$, it is clear that the mode $j=m=3$ is the dominant superradiant one, a result that was also found in the massless case [30]. The suppression of the superradiance with the number of extra dimensions observed in [30] for massless bulk scalar modes holds also in the massive case, and thus we do not comment further. A feature that has not been noted before is the non-monotonic behaviour of both the magnitude of the superradiance effect and the extent of the superradiant regime in terms of the angular-momentum parameter $a_{*}$ : in Fig. 3(c), we see that, as $a_{*}$ increases from zero to 1.5, the superradiance effect is indeed enhanced, however, this behaviour is reversed when $a_{*}$ increases further. In addition, 

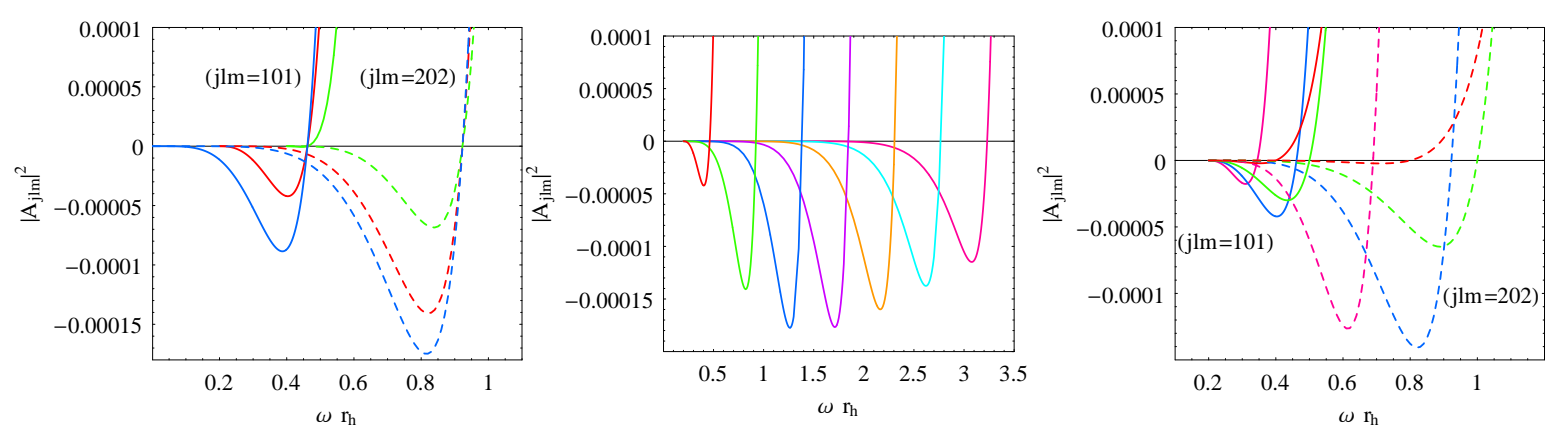

Figure 3: The dependence of superradiance for bulk massive scalar modes on (a) the mass, for $a_{*}=1.5, n=2$ and $m_{\Phi}=0,0.2,0.4$ (from bottom to top), (b) on the angular momentum numbers, for $a_{*}=1.5, n=2, m_{\Phi}=0.2$ and $j=m=1,2,3,4,5,6,7$ (from left to right), and (c) on the angular momentum parameter, for $n=2, m_{\Phi}=0.2$ and $a_{*}=0.5$ (red), 1 (green), 1.5 (blue), 2.5 (magenta).

superradiance occurs for frequencies $m_{\Phi}<\omega<\omega_{s}=m a /\left(r_{h}^{2}+a^{2}\right)$ : the latter restriction is imposed by the vanishing of the value of the absorption probability; the former by the demand that its value is a real number, and signifies the fact that no particles of mass $m_{\Phi}$ can be created if energy less than that is available. Interestingly enough the width of the superradiance regime, $\delta \omega=m a /\left(r_{h}^{2}+a^{2}\right)-m_{\Phi}$, does not monotonically grow with the increase of the angular momentum of the black hole, as one could instictively expect. Indeed, its value reaches a maximum for a particular value of the angular-momentum parameter, namely $a= \pm r_{h}$, which is in fact independent of the mass and angular momentum numbers of the mode as well as of the number of extra dimensions 1 . For the case depicted in Fig. 33(c), where we have fixed the horizon value at $r_{h}=1$ and considered only positive values of $a_{*}$, the superradiant regime takes its maximum value at $a_{*}=1$, beyond which it starts to shrink, for both modes $(j \ell m=101)$ and $(j \ell m=202)$.

\subsection{Energy Emission Rate in the Bulk}

We will next compute the rate of energy emission in the bulk in the form of massive scalar fields by using the exact numerical results for the absorption probability found in the previous section. The emission of energy per unit frequency and unit time in the bulk is given by the expression [33, 29, 30]

$$
\frac{d^{2} E}{d t d \omega}=\frac{1}{2 \pi} \sum_{j, \ell, m} \frac{\omega}{\exp \left[k / T_{\mathrm{H}}\right]-1} N_{\ell}\left|\mathcal{A}_{j \ell m}\right|^{2} .
$$

The multiplicity of states $N_{\ell}$ from the expansion of the wavefunction of the field in the $n$-dimensional sphere is given in Eq. (35) and the parameter $k$ is defined in Eq. (17).

\footnotetext{
${ }^{1}$ The monotonic behaviour of the width and depth of the superradiance regime found in 29 is not in contradiction with the results found here as only low values of the angular momentum of the black hole, lower than the turning points found here and in agreement with the low- $a_{*}$ approximation used in [29, were considered in there.
} 

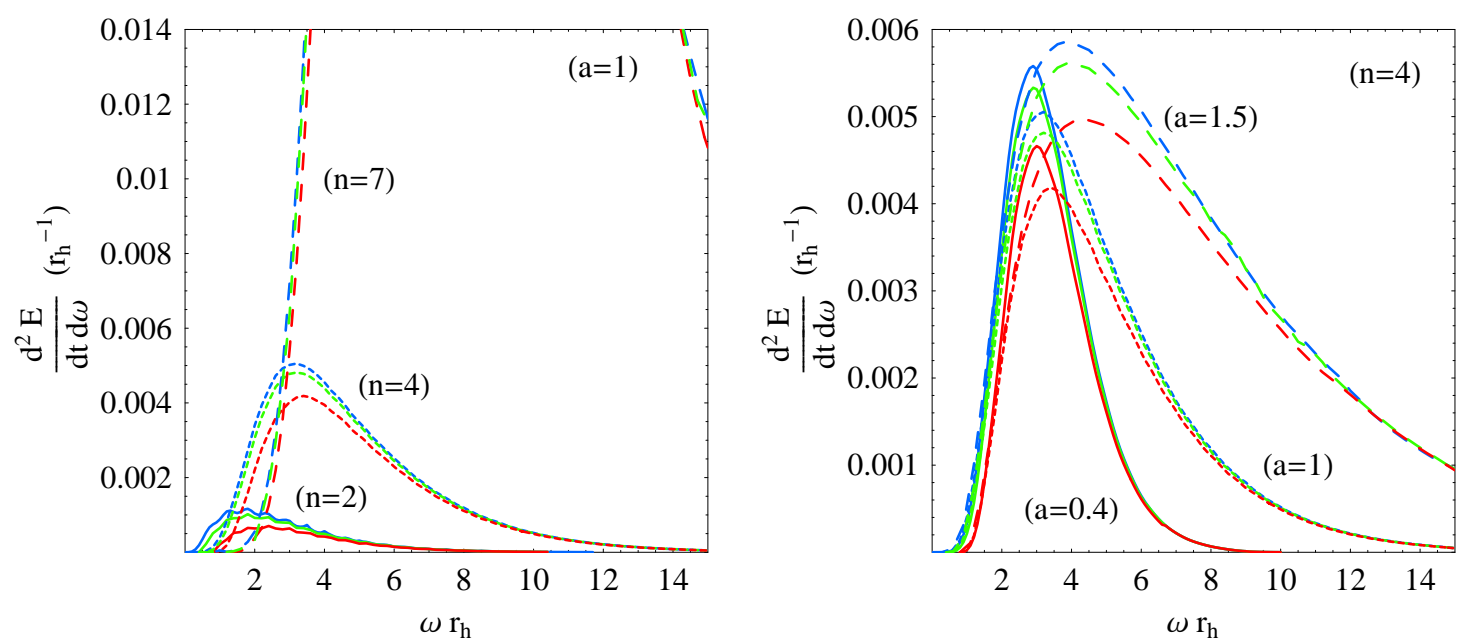

Figure 4: Energy emission rates for bulk scalar fields for: (a) for $a_{*}=1, n=2,4,7$ and $m_{\Phi}=0,0.4,0.8$ (from top to bottom in each set of curves with fixed $n$ ), and (b) $n=4$, $a_{*}=0.4,1,1.5$ and $m_{\Phi}=0,0.4,0.8$ (from top to bottom in each set of curves with fixed $\left.a_{*}\right)$.

Finally, the temperature of the higher-dimensional, simply-rotating black hole (10) is

$$
T_{\mathrm{H}}=\frac{(n+1)+(n-1) a_{*}^{2}}{4 \pi\left(1+a_{*}^{2}\right) r_{h}} .
$$

Equation (38) is identical in form with the expression for the emission of massless scalar fields in the bulk, nevertheless, there are two major differences: the calculation of the spectrum starts from $\omega=m_{\Phi}$ instead of zero, and the value of the absorption probability depends, apart from the spacetime parameters, on the characteristics of the emitted field including its mass.

In order to derive the energy emission spectrum, we need to sum over a significantly large number of partial waves labeled by the set of $(j, \ell, m)$ quantum numbers. For each value of $n, a_{*}$ and $m_{\Phi}$, we aimed at deriving the complete spectrum, i.e. to reach values of the energy parameter $\omega r_{h}$ where the corresponding value of the energy emission rate would be less than $10^{-6}$. At the same time, the number of partial waves summed had to be large enough so that the derivation of the energy spectrum would be as close as possible to the real one - especially for the computation of the total emissivity presented in section 4. Taking all these constraints into account, we were able to sum the contribution of all bulk scalar modes up to $j=30$, that brings the total number of summed modes to $N_{\mathrm{bu}}=5456$. According to our estimates, the contribution of all modes higher than $j=30$ should be less than $5 \%$, for the higher values of parameters considered, namely $n=7$ and $a_{*}=1.5$, an error that falls below $0.001 \%$ for the lowest values considered, i.e. $n=2$ and $a_{*}=0.4$.

In Fig. 4, we depict the energy emission rate on the brane in the form of massive scalar fields in terms of the number of extra dimensions, value of the angular-momentum 
parameter, and mass of the emitted field itself. Thus, Fig. 4(a) shows the energy emission rate for fixed $a_{*}\left(a_{*}=1\right)$ and variable $n=2,4,7$ and $m_{\Phi}=0,0.4,0.8$, while Fig.4(b) plots the same quantity but for fixed $n(n=4)$ and variable $a_{*}=0.4,1,1.5$. In terms of the spacetime parameters $n$ and $a_{*}$, these plots confirm the behaviour found in the case of massless fields [29, 30]: the power spectrum is enhanced as the number of extra dimensions increases while its dependence on the angular momentum parameter is not monotonic but differs as $n$ and/or $\omega$ varies. More detailed features, like the oscillatory pattern of the emission curves for low values of $n$ and $a_{*}$, that are replaced by more smoother curves as the values of these parameters increase, are also recovered.

In terms of the mass of the scalar field, we observe the expected suppression of the emission rate, for fixed $n$ and $a_{*}$, as $m_{\Phi}$ increases - the suppression is more prominent in the low- and intermediate-energy regimes whereas the effect of the mass becomes negligible at the high-energy regime. Compared to the case of the emission of masless scalar fields, the suppression in the low-energy regime becomes even more significant if the disappareance of the frequency range with $\omega<m_{\Phi}$ is taken into account. The magnitude of the suppression with $m_{\Phi}$ depends strongly on the particular value of $n$ and $a_{*}$ - the exact effect will be computed in section 4 where the total emissivities in bulk and brane will be calculated.

\section{Emission of Massive Scalars on the Brane}

In this section, we turn our attention to the emission of massive scalar fields by a higherdimensional simply-rotating black hole on the brane. The analysis for the derivation of the absoprtion probability, both analytical and numerical, is quite similar to the one performed for the emission in the bulk; aspects of it have also been recently addressed in a set of publications [34] that appeared while this work was still in progress. For the sake of comparison and completeness of the analysis, we will still present in this section the most important points of our calculation on the brane and focus our discussion to aspects not covered before; these include, for example, the analytic study of the lowenergy asymptotic behaviour of the absorption probability and cross-section, the role of the angular momentum of the black hole, that was ignored in [34], and the form of the energy emission spectrum, instead of the number flux that was studied in the same work.

Let us start with the form of the gravitational background that a massive scalar field sees as it propagates on the brane and its corresponding field equation. The 4-dimensional induced background will be the projection of the higher-dimensional one (11) onto the brane, and follows by fixing the values of the angular variables of the $n$-sphere. Then, the induced-on-the-brane line-element takes the form

$$
\begin{aligned}
d s^{2}=\left(1-\frac{\mu}{\Sigma r^{n-1}}\right) d t^{2}+\frac{2 a \mu \sin ^{2} \theta}{\Sigma r^{n-1}} d t d \varphi-\frac{\Sigma}{\Delta} d r^{2} \\
-\Sigma d \theta^{2}-\left(r^{2}+a^{2}+\frac{a^{2} \mu \sin ^{2} \theta}{\Sigma r^{n-1}}\right) \sin ^{2} \theta d \varphi^{2},
\end{aligned}
$$

which is very similar to the usual 4-dimensional Kerr one but carries an explicit depen- 
dence on the number of additional spacelike dimensions $n$. The field equation is still given by the covariant form (44) but with the higher-dimensional metric tensor $G_{M N}$ replaced by the 4-dimensional one $g_{\mu \nu}$ defined above. The field factorization

$$
\Phi(t, r, \theta, \varphi)=e^{-i \omega t} e^{i m \varphi} P(r) T(\theta)
$$

leads again to the decoupling of variables and to the following set of radial and angular equations

$$
\begin{gathered}
\frac{d}{d r}\left(\Delta \frac{d P}{d r}\right)+\left(\frac{K^{2}}{\Delta}-\tilde{\Lambda}_{j m}-m_{\Phi}^{2} r^{2}\right) P=0, \\
\frac{1}{\sin \theta} \frac{d}{d \theta}\left(\sin \theta \frac{d T}{d \theta}\right)+\left(\tilde{\omega}^{2} a^{2} \cos ^{2} \theta-\frac{m^{2}}{\sin ^{2} \theta}+\tilde{E}_{j m}\right) T=0,
\end{gathered}
$$

respectively. In the above, we have defined $\tilde{\Lambda}_{j m}=\tilde{E}_{j m}+a^{2} \omega^{2}-2 a m \omega$, while $\tilde{\omega}$ is again given by $\tilde{\omega}=\sqrt{\omega^{2}-m_{\Phi}^{2}}$ and $K$ by Eq. (10). The angular function $T(\theta)$ satisfies again a modified spheroidal harmonics equation with $\omega \rightarrow \tilde{\omega}$. The corresponding massive eigenvalue $\tilde{E}_{j m}(a \tilde{\omega})$ is thus related to the massless one through the same shift, and in terms of a power series [43] is given by

$$
\begin{aligned}
\tilde{E}_{j m} & =j(j+1)+(a \tilde{\omega})^{2} \frac{\left[2 m^{2}-2 j(j+1)+1\right]}{(2 j-1)(2 j+3)} \\
& +(a \tilde{\omega})^{4}\left\{\frac{2\left[-3+17 j(j+1)+j^{2}(j+1)^{2}(2 j-3)(2 j+5)\right]}{(2 j-3)(2 j+5)(2 j+3)^{3}(2 j-1)^{3}}\right. \\
& +\frac{4 m^{2}}{(2 j-1)^{2}(2 j+3)^{2}}\left[\frac{1}{(2 j-1)(2 j+3)}-\frac{3 j(j+1)}{(2 j-3)(2 j+5)}\right] \\
& \left.+\frac{2 m^{4}[48+5(2 j-1)(2 j+3)]}{(2 j-3)(2 j+5)(2 j-1)^{3}(2 j+3)^{3}}\right\}+\mathcal{O}\left((a \tilde{\omega})^{6}\right),
\end{aligned}
$$

The above form will be used in the computation of the absorption probability both analytically and numerically.

\subsection{The Absorption Probability on the Brane}

The approximation method employed in section 2 can again be used to solve the radial equation (42) analytically. The same change of variable $r \rightarrow f(r)=\Delta(r) /\left(r^{2}+a^{2}\right)$, in the near-horizon regime $\left(r \simeq r_{h}\right)$, leads to an equation of the form (12) where now

$$
D_{*} \equiv 1+\frac{n\left(1+a_{*}^{2}\right)}{A_{*}}-\frac{4 a_{*}^{2}}{A_{*}^{2}}, \quad C_{*} \equiv\left(\tilde{\Lambda}_{j m}+m_{\Phi}^{2} r_{h}^{2}\right)\left(1+a_{*}^{2}\right),
$$

while $A_{*}$ and $K_{*}$ are defined as in the bulk. The field redefinition $P(f)=f^{\alpha}(1-f)^{\beta} F(f)$ reduces the above differential equation to a hypergeometric one with the physically acceptable solution in the near-horizon regime given by

$$
P_{N H}(f)=A_{-} f^{\alpha}(1-f)^{\beta} F(a, b, c ; f) .
$$


In the above, $A_{-}$is again an arbitrary integration constant, and $a=\alpha+\beta+D_{*}-1$, $b=\alpha+\beta, c=1+2 \alpha$. The power coefficients $\alpha$ and $\beta$ are given by the expressions in Eq. (14), with $D_{*}$ and $C_{*}$ now taken their brane values. Under the choice $\alpha=\alpha_{-}$, that we will henceforth use, the above solution reduces, as expected, to an ingoing plane wave, $P_{N H} \simeq A_{-} f^{-i K_{*} / A_{*}}=A_{-} e^{-i k y}$ with $k$ defined in Eq. (17).

In the far-field regime $\left(r \gg r_{h}\right)$, the radial equation (42), under the substitution $P(r)=\frac{1}{\sqrt{r}} \tilde{P}(r)$, takes again the form of a Bessel differential equation leading to the general solution

$$
P_{F F}(r)=\frac{B_{1}}{\sqrt{r}} J_{\nu}(\tilde{\omega} r)+\frac{B_{2}}{\sqrt{r}} Y_{\nu}(\tilde{\omega} r)
$$

where now $\nu=\sqrt{\tilde{E}_{j m}+a^{2} \tilde{\omega}^{2}+1 / 4}$.

The process of the matching proceeds as in the case of bulk emission. The nearhorizon solution (46), after it is shifted, is expanded in the limit $r \gg r_{h}$, while the far-field one (47) is expanded in the $r \rightarrow 0$ limit. Both reduce to polynomial forms similar to those in Eqs. (20) and (22). If we again ignore terms of order $\left(\tilde{\omega}_{*}^{2}, a_{*}^{2}, a_{*} \tilde{\omega}_{*}\right)$ or higher in the power coefficients, we obtain $-(n+1) \beta \simeq j,(n+1)\left(\beta+D_{*}-2\right) \simeq-(j+1)$, and $\nu \simeq j+1 / 2$. These simplifications hold provided that the mass of the scalar field on the brane does not exceed an upper value: following a similar argument to the case of the bulk emission, this constraint is found to be $m_{\Phi}<(250-500) \mathrm{GeV}$ for $n=1-7$ - note that the upper value of the mass on the brane is reduced by a factor of two compared to the one in the bulk. Then, the matching of the corresponding multiplicative in the coefficients, leads to the constraint

$$
\begin{aligned}
B \equiv \frac{B_{1}}{B_{2}}= & -\frac{1}{\pi}\left(\frac{2}{\tilde{\omega} r_{h}\left(1+a_{*}^{2}\right)^{\frac{1}{n+1}}}\right)^{2 j+1} \sqrt{\tilde{E}_{j m}+a^{2} \tilde{\omega}^{2}+1 / 4} \\
& \times \frac{\Gamma^{2}\left(\sqrt{\tilde{E}_{j m}+a^{2} \tilde{\omega}^{2}+1 / 4}\right) \Gamma\left(\alpha+\beta+D_{*}-1\right) \Gamma(\alpha+\beta) \Gamma\left(2-2 \beta-D_{*}\right)}{\Gamma\left(2 \beta+D_{*}-2\right) \Gamma\left(2+\alpha-\beta-D_{*}\right) \Gamma(1+\alpha-\beta)} .
\end{aligned}
$$

The above completes the derivation of the analytic solution for the radial part of the massive scalar field on the brane. By expanding the far-field solution (47) at asymptotic infinity, we recover again a superposition of spherical waves

$$
P_{F F}(r) \simeq \frac{1}{\sqrt{2 \pi \tilde{\omega}}}\left[\frac{\left(B_{1}+i B_{2}\right)}{r} e^{-i\left(\tilde{\omega} r-\frac{\pi}{2} \nu-\frac{\pi}{4}\right)}+\frac{\left(B_{1}-i B_{2}\right)}{r} e^{i\left(\tilde{\omega} r-\frac{\pi}{2} \nu-\frac{\pi}{4}\right)}\right] .
$$

The absorption probability for the brane emission $\left|\mathcal{A}_{j m}\right|^{2}$ is then given again by the right-hand-part of Eq. (27) with $B$ in this case defined in Eq. (48).

At the very low-energy regime, we may again derive a simplified, compact expression for the absorption probability. Following the same analysis as in the case of bulk emission, we obtain $\left|\mathcal{A}_{j m}\right|^{2}=\Sigma_{1} \times \Sigma_{2} \times \Sigma_{3}$, where

$$
\Sigma_{1}=\frac{-2 i \pi\left(\tilde{\omega} r_{h} / 2\right)^{2 j+1}}{\left(j+\frac{1}{2}\right) \Gamma^{2}\left(j+\frac{1}{2}\right)} \frac{\left(1+a_{*}^{2}\right)^{\frac{2 j+1}{n+1}} \Gamma\left(2 \beta+D_{*}-2\right)}{\Gamma\left(2-2 \beta-D_{*}\right)},
$$


while $\Sigma_{2}$ and $\Sigma_{3}$ are given by the corresponding bulk equations (30) and (31) but with the parameters $\alpha, \beta$ and $D_{*}$ now taken their brane values. For modes with $m>0$, the limit $\alpha \rightarrow 0$, will give us the behaviour of the absorption probability at the upper boundary of the superradiance regime which is given by

$$
\left|\mathcal{A}_{j m}\right|^{2}=\frac{4 \pi\left(\tilde{\omega} r_{h} / 2\right)^{2 j+1} K_{*} \sin ^{2} \pi\left(2 \beta+D_{*}\right) \Gamma^{2}\left(2 \beta+D_{*}-2\right) \Gamma^{2}(1-\beta)\left(2-D_{*}-2 \beta\right)}{A_{*}\left(1+a_{*}^{2}\right)^{-\frac{2 j+1}{n+1}}\left(j+\frac{1}{2}\right) \Gamma^{2}\left(j+\frac{1}{2}\right) \Gamma^{2}\left(\beta+D_{*}-1\right) \sin ^{2} \pi\left(\beta+D_{*}\right)} .
$$

As expected it is again the sign of $K_{*}$ that defines the sign of the absorption probability in this energy regime since $K_{*}=r_{h}\left(1+a_{*}^{2}\right)\left(\omega-m \Omega_{h}\right)$. By setting $j=m=0$ and expanding further in the limit $\omega \rightarrow 0$, Eq. (51) can also give us the asymptotic value of $\left|\mathcal{A}_{00}\right|^{2}$ for the dominant scalar mode, which is

$$
\left|\mathcal{A}_{00}\right|^{2}=\frac{4 \omega \tilde{\omega} r_{h}^{2}\left(1+a_{*}^{2}\right)}{A_{*}\left(1+a_{*}^{2}\right)^{-1 /(n+1)}\left(2-D_{*}\right)}+\ldots .
$$

Equation (34) may also provide the relation between the absorption cross-section and the absoprtion probability for a massive scalar field living on the brane. By setting $n=0$ and $N_{\ell}=1$, since the brane modes do not 'see' the $n$-sphere, we obtain the 4 -dimensional formula

$$
\sigma_{00}=\frac{\pi}{\tilde{\omega}^{2}}\left|\mathcal{A}_{0}\right|^{2}=4 \pi\left(\frac{\omega}{\tilde{\omega}}\right)\left(r_{h}^{2}+a^{2}\right) \frac{\left(1+a_{*}^{2}\right)^{1 /(n+1)}}{\left[(n+1)+(n-1) a_{*}^{2}\right]\left(2-D_{*}\right)}+\ldots
$$

Again, for $m_{\Phi}=0$ and $a_{*}=0$, the value of the absorption cross-section reduces to the area $4 \pi r_{h}^{2}$ of the 4 -dimensional Schwarzschild black hole, as expected [7, 8]; for $a_{*} \neq 0$, it approaches the area $4 \pi\left(r_{h}^{2}+a^{2}\right)$ of the 4-dimensional rotating black hole for small values of the angular-momentum parameter [27]. However, as soon as the mass of the scalar field becomes larger than zero, the aforementioned constant values of $\sigma_{00}$ are replaced by diverging ones for both rotating and non-rotating black holes - in the latter case, this is again in accordance with previous analyses [33, 23]. As in the case of the bulk scalar field, and due to the $\tilde{\omega}^{2 j+1}$ factor in Eq. (51), all higher modes with $j \geq 1$ have a vanishing asymptotic value as $\tilde{\omega} \rightarrow 0$.

The derivation of the complete energy spectrum demands once again the calculation of the value of the absorption probability by numerical means. The asymptotic behaviour of the brane massive scalar field close to and far away from the black hole horizon is similar to the one of a bulk field: it is an incoming plane wave in the near-horizon regime, as discussed below Eq. (46), and a spherical wave at radial infinity according to Eq. (47). The numerical integration of the radial differential equation (42) on the brane is performed by using the same method as in the bulk: the integration starts very close to the black-hole horizon with boundary conditions given again by Eq. (37) and proceeds until we reach radial infinity, where the amplitudes of the incoming and outgoing modes are isolated to compute the value of the absorption probability $\left|\mathcal{A}_{j m}\right|^{2}$.

Therefore, in Fig. 5, we depict the exact numerical results for the value of the absorption probability of the dominant mode $j=m=0$. In Fig. 5)(a), the value of $\left|\mathcal{A}_{00}\right|^{2}$ is plotted for fixed angular momentum $\left(a_{*}=1\right)$ and variable $m_{\Phi}$, equal to $0,0.4,0.8$ 

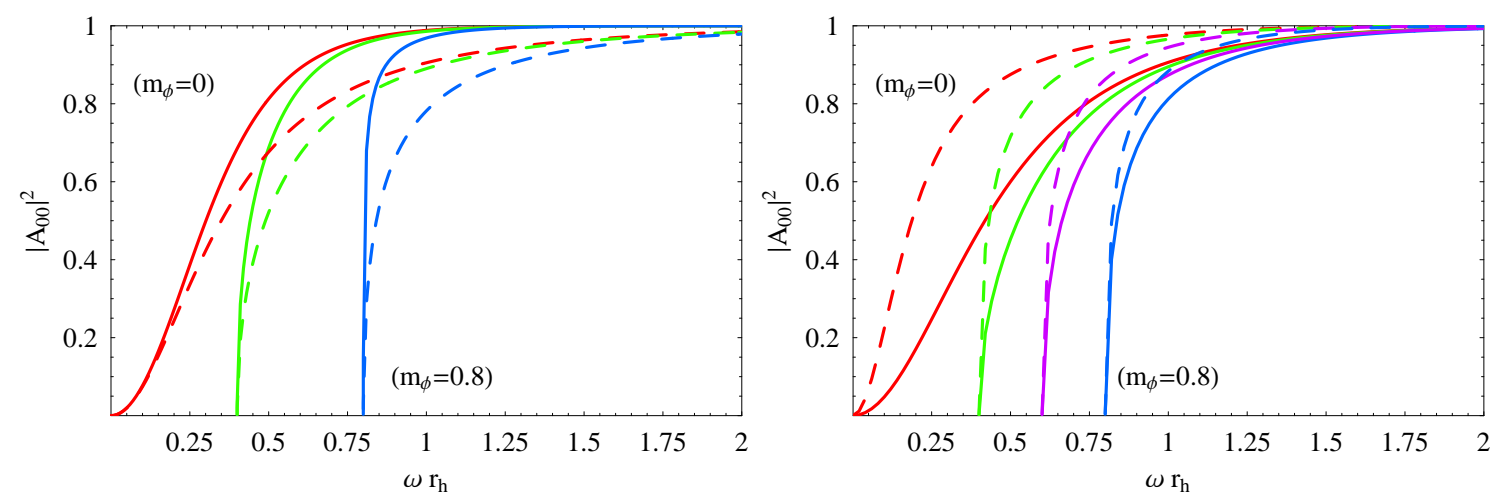

Figure 5: Absorption probabilities for the brane scalar mode $j=m=0$, for (a) $a_{*}=1, m_{\Phi}=0,0.4,0.8$ and $n=2$ (solid lines) and 7 (dashed lines), and (b) $n=4$, $m_{\Phi}=0,0.4,0.6,0.8$ and $a_{*}=0.5$ (solid lines) and 2.5 (dashed lines).

(from left to right), and for two different values of the number of extra dimensions, $n=2$ (solid lines) and $n=7$ (dashed lines). As expected, the value of the absorption probability is suppressed with the number of extra dimensions, as noted before in the literature [20, 24, 27]. The suppression becomes significantly more important as the value of the mass of the brane scalar field increases - this is also in agreement 2 with the results derived recently in [34, therefore, we do not comment on this further. On the other hand, Fig. 5. (b) depicts the dependence of $\left|\mathcal{A}_{00}\right|^{2}$ on the angular-momentum parameter, that takes the values $a_{*}=0.5$ and $a_{*}=2.5$, while $n$ remains fixed $(n=4)$ and $m_{\Phi}$ changes from 0 to 0.8 (from left to right again). For $m_{\Phi}=0$, the absorption probability increases as $a_{*}$ increases, too, in accordance again with the literature [20, 24, 27] - the same behaviour is observed as the mass of the scalar field becomes larger but with the enhancement becoming increasingly less significant. For the purpose of the analysis presented in section 4, where the bulk and brane energy spectra are compared, let us note here that both effects, the suppression with $n$ and the enhancement with $a_{*}$, are much more prominent for massive bulk scalar fields than for brane fields of the same type.

As in the case of the emission in the bulk, we would like to investigate the validity of the analytic method used above to derive the value of the absorption probability for the emission of massive scalar fields on the brane, and how this is affected by the value of the mass and angular-momentum numbers of the emitted field, the number of extra dimensions and the magnitude of the angular momentum of the black hole. To this end, in Fig. 6(a), we plot both the analytical (solid lines) and numerical (data points) results for the absorption probability of the indicative mode $(j, m)=(1,-1)$, for fixed angularmomentum parameter $\left(a_{*}=1\right)$ and number of extra dimensions $(n=4)$ and $m_{\Phi}=$ $0,0.4,0.6,0.8$. We observe that the agreement between the two sets of results remains

\footnotetext{
${ }^{2}$ The agreement is mainly qualitative as our results are derived for non-vanishing angular momentum parameter $a_{*}$ while in [34] the effect of the rotation of the black hole was ignored and the role of the mass and charge of the brane field was studied instead. Nevertheless, there is a general agreement between the two sets of results in terms of both the number of extra dimensions and the value of the mass of the brane scalar field.
} 

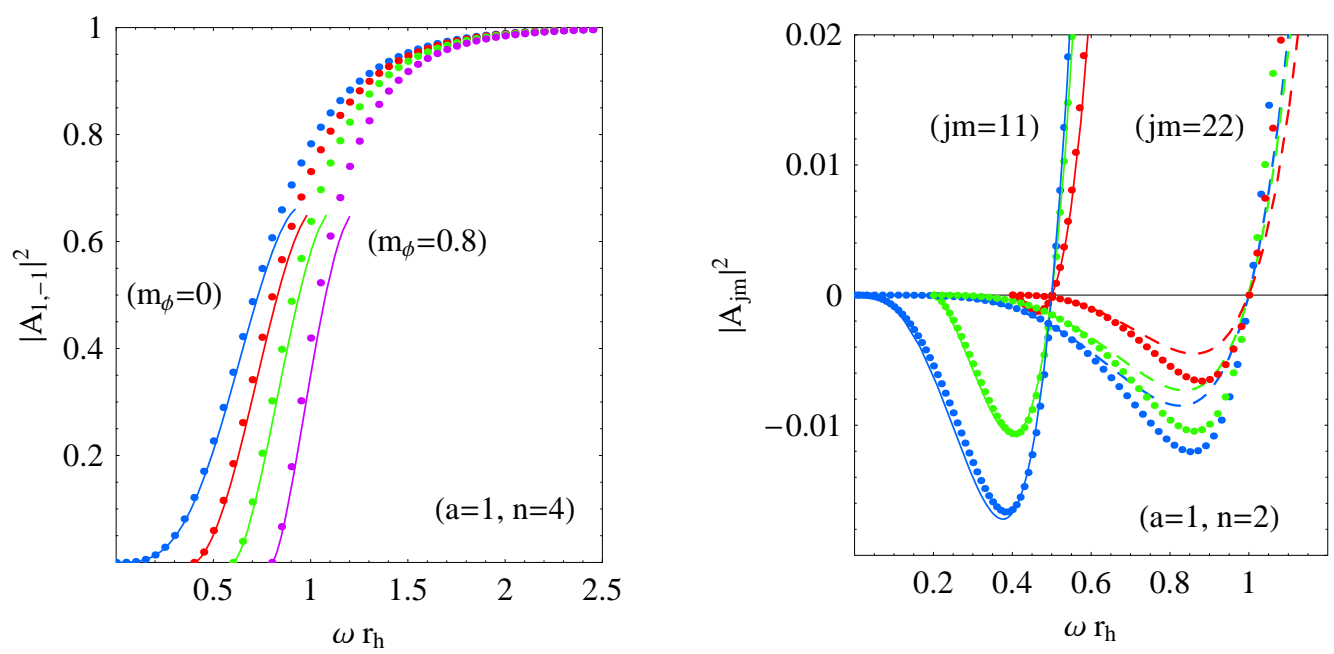

Figure 6: Comparison of the analytical (solid lines) and exact numerical (data points) results for the absorption probability for: (a) the brane scalar mode $(j, m)=(1,-1)$, for $a_{*}=1, n=4$ and $m_{\Phi}=0,0.4,0.6,0.8$, and (b) the superradiant brane scalar modes $(j, m)=((1,1),(2,2))$ for $a_{*}=1, n=2$, and $m_{\Phi}=0,0.2,0.4$ (from bottom to top).

particularly good even well beyond the low-energy regime. The solid lines terminate again due to the existence of poles in the arguments of the Gamma functions in the analytic expression of the absorption coefficient. We find that the appearance of the poles, for modes with given $j$, takes place much earlier for the modes with $m=0$ than for $m>0$, while for the ones with $m<0$ this happens at much higher values of the energy, a fact which significantly extends the range of validity of the analytic results in the latter case as is clear from Fig. 6(a). In Fig. 6(b), we focus on the low-energy regime of two superradiant modes, $(j, m)=(1,1)$ and $(j, m)=(2,2)$ : in agreement with results drawn in the massless case [27], we find that the analytic results for the value of the absorption probability start to deviate from the exact numerical ones as the angularmomentum numbers of the mode increase; this is due to the shift of the curve towards higher values of the energy - note that the range of agreement extends well beyond the value $\omega r_{h}=0.6$ for both modes, however, for the $(j, m)=(2,2)$ mode this covers only a part of the superradiant regime contrary to what happens for the $(j, m)=(1,1)$ mode. It deserves to be noted that the value of the mass of the emitted field affects the relative values of the absorption probability of different superradiant modes: while for $m_{\Phi}=0$, the $(j, m)=(1,1)$ mode dominates over the $(j, m)=(2,2)$ one, this radically changes as soon as the mass of the scalar field exceeds the value $m_{\Phi}=0.2$.

Let us finally comment on the range of validity of the analytic results in terms of the parameters of the higher-dimensional spacetime. In Fig. 7(a), we plot both sets of results for the mode $(j, m=1,-1)$ for fixed angular-momentum parameter $\left(a_{*}=1\right)$ and mass of the field $\left(m_{\Phi}=0.4\right)$ while the number of extra dimensions takes the values $n=2,4,7$. From the plot, it is clear that the agreement between the analytic and numerical results is excellent for low values of $n$ while it quickly worsens as the number 

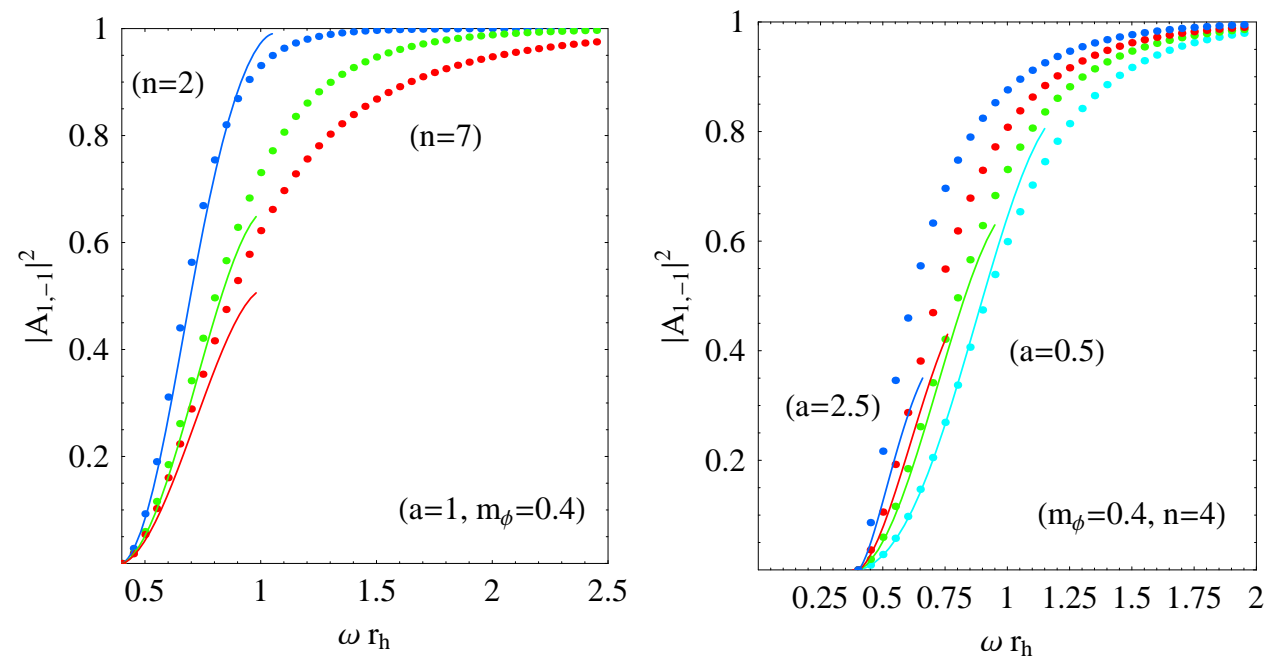

Figure 7: Comparison of the analytical (solid lines) and exact numerical (data points) results for the absorption probability for the brane scalar mode $(j, m=1,-1)$, for (a) $a_{*}=1, m_{\Phi}=0.4$ and $n=2,4,7$, and (b) $m_{\Phi}=0.4, n=4$ and $a_{*}=0.5,1,1.5,2.5$.

of extra dimensions increases. Figure 7 (b) plots the two sets of results for the same mode for fixed mass $\left(m_{\Phi}=0.4\right)$ and number of extra dimensions $(n=4)$, but variable angularmomentum parameter $\left(a_{*}=0.5,1,1.5,2.5\right)$. Again, the agreement extends well beyond the intermediatate-energy regime for low values of $a_{*}$ while it is gradually restricted in the low-energy regime as the value of $a_{*}$ increases. The observed behaviour is in agreement with the one found in the massless case [27] and stems from the fact that several of our approximations in the analytic method become less accurate as either $n$ or $a_{*}$ increases.

\subsection{Energy Emission Rate on the Brane}

The exact value of the absorption probability $\left|\mathcal{A}_{j m}\right|^{2}$ for massive scalar fields on the brane, as this followed after the numerical integration of the radial equation of the wavefunction, will now be used for the computation of the corresponding energy emission rate. The higher-dimensional, simply-rotating black hole emits massive scalar particles on the brane with a rate given by the exression [18, 20, 21, 24]

$$
\frac{d^{2} E}{d t d \omega}=\frac{1}{2 \pi} \sum_{j, m} \frac{\omega}{\exp \left[k / T_{\mathrm{H}}\right]-1}\left|\mathcal{A}_{j m}\right|^{2} .
$$

In the above, $k$ is defined in Eq. (17) as before, while the temperature for the emission on the brane is that of the higher-dimensional black hole given in Eq. (39). As in the case of bulk emission, the formula of the emission rate for massive fields is the same as the one for massless, with the effect of the mass being encoded in the value of the absoprtion probability and the frequency range of the emission.

As in the case of the bulk emission, for the derivation of the energy emission spectrum on the brane we need to sum over a significantly large number of partial waves labeled 

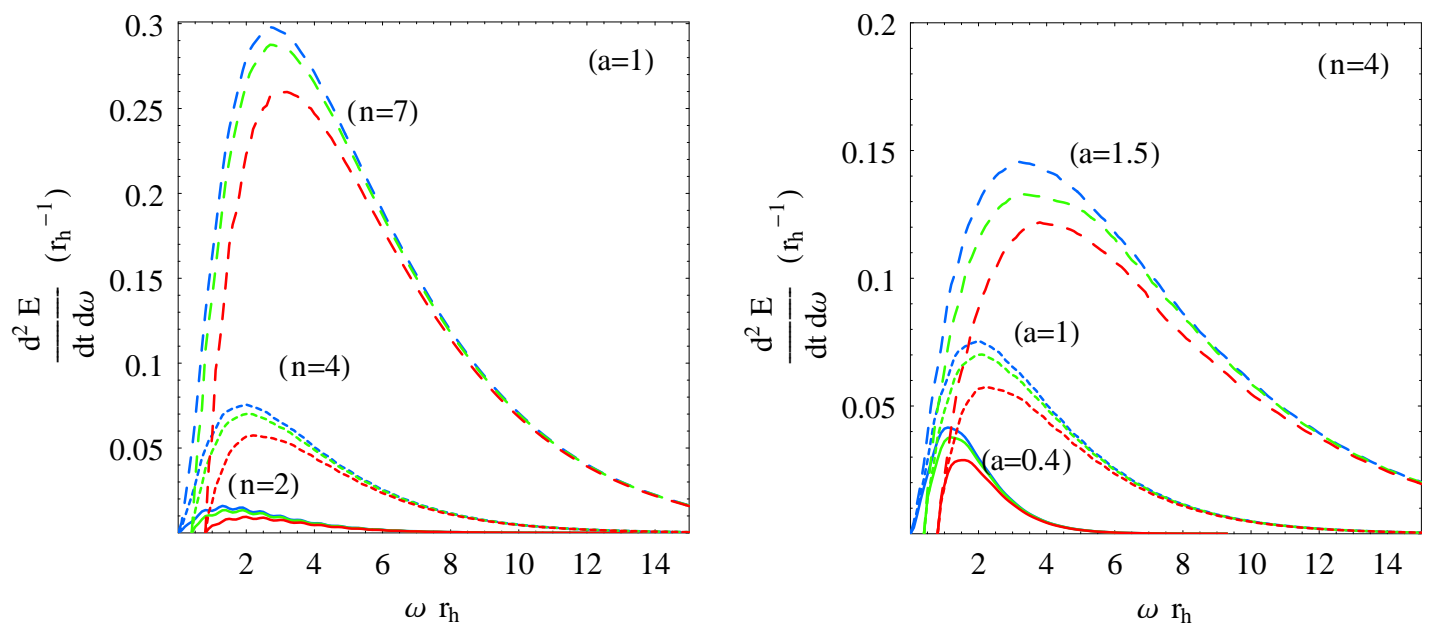

Figure 8: Energy emission rates for brane scalar fields for: (a) for $a_{*}=1, n=2,4,7$ and $m_{\Phi}=0,0.4,0.8$ (from top to bottom in each set of curves with fixed $n$ ), and (b) $n=4$, $a_{*}=0.4,1,1.5$ and $m_{\Phi}=0,0.4,0.8$ (from top to bottom in each set of curves with fixed $\left.a_{*}\right)$.

by the $(j, m)$ quantum numbers. The absence of the 'internal' quantum number $\ell$, that further characterizes the bulk modes, makes the brane summation easier, nevertheless the process remained significantly time-consuming 3 . We summed the contribution of all modes up to $j=40$, that brings the total number of brane modes to $N_{b r}=1681$, and computed the spectrum up to the value of energy where the power rate dropped again below $10^{-6}$. According to our estimates, the error in our results by leaving out the higher modes is less than $5 \%$, for the higher values of $n$ and $a_{*}$ considered, and below $0.001 \%$ for the lowest.

In Fig. 8, we plot the energy emission rate on the brane in the form of massive scalar fields in terms of the number of extra dimensions, value of the angular-momentum parameter, and mass of the emitted field - we have kept the same values of these parameters as in the case of bulk emission for easier comparison. Figure 8(a) shows the energy emission rate for fixed $a_{*}\left(a_{*}=1\right)$ and variable $n=2,4,7$ and $m_{\Phi}=0,0.4,0.8$, while in Fig. 8(b) we keep $n$ fixed $(n=4)$ and vary $a_{*}=0.4,1,1.5$. Again, our results reproduce succesfully the behaviour found in the case of massless fields [20, 24, 29], and demonstrate that the enhancement of the emission spectrum as either the number of extra dimensions or the angular momentum of the black hole increases persists even for non-vanishing values of the mass of the emitted field. The mass of the scalar field causes again the suppression of the spectrum in all energy regimes, apart from the very high-energy one where its effect becomes negligible. The suppression is again strongly dependent on the particular value of $n$ and $a_{*}$. By comparing Figs. 4 and 8 , we see that the brane emission is larger

\footnotetext{
${ }^{3}$ For the largest values of the parameters considered, i.e. $n=7$, and $a_{*}=1.5$, the derivation of the complete spectrum for each value of the mass $m_{\Phi}$ lasted more than 4 days - this is to be contrasted with the corresponding summation in the bulk where a single run lasted more than 6 days.
} 
than the bulk emission by more than an order of magnitude - accordingly, we expect the suppression with $m_{\Phi}$ to be larger on the brane than in the bulk. The exact role of $m_{\Phi}$ in the total emissivity of the black hole, in conjunction with the parameters $\left(n, a_{*}\right)$ and the type of emission channel (brane or bulk), will be investigated in the next section.

\section{Bulk and Brane Total Emissivities}

Although the global properties of the absorption probability and energy spectra do not change when the mass of the scalar field is introduced, important variations in their values appear which differ as the value of $m_{\Phi}$, together with that of either $n$ or $a_{*}$, changes. For this reason, we expect that differences will appear when the spectra for the emission of massive and massless fields are compared. These differences may be evident at particular energy regimes or range of values of the parameters $\left(n, a_{*}\right)$, and may significantly affect the total energy emissivities. The modifications in the spectrum may also be different when bulk or brane emission is considered, therefore, in this section we compute the total emissivities for both emission channels and compare them.

To this end, we have integrated the differential energy rates per unit time and unit frequency, computed in sections 2 and 3, over the entire frequency range of emission. In Tables 1 and 2, we present the corresponding total emissivities for bulk and brane emission, respectively, for some indicative values of the number of extra dimensions $(n=2,4,7)$, angular-momentum parameter of the black hole $\left(a_{*}=0.4,1,1.5\right)$ and mass of the emitted field $\left(m_{\Phi}=0,0.4,0.8\right)$. The values of the total emissivities are normalised to the one for $n=2, a_{*}=0.4$ and $m_{\Phi}=0$, in each case, for easy comparison.

The entries of both tables confirm the enhancement of the total emissivities as either $n$ or $a_{*}$ increases and the suppression with $m_{\Phi}$. As it was anticipated from the plots, the suppression is strongly dependent not only on $m_{\Phi}$ but also on both $n$ and $a_{*}$. We obsereve that, as either $n$ or $a_{*}$ increases, the suppression of the total emissivity with the mass of the scalar field decreases in magnitude. Starting from the bulk channel (Table 1 ), we see that for a fixed, low value of $n$, i.e. $n=2$ the total emission for a scalar field with mass $m_{\Phi}=0.8$ drops to $52 \%$ of the emission for a massless field, if $a_{*}=0.4$, but to $71 \%$ if $a_{*}=1.5$. The suppression is even more limited when the value of $n$ takes a much higher value: thus, for $n=7$, the emission for a $m_{\Phi}=0.8$ scalar field in the bulk drops only to the $92 \%$ of the massless value if $a_{*}=0.4$ and to $95 \%$ if $a_{*}=1.5$. It seems that both the number of extra dimensions and the rotation of the black hole subsidize the emission of massive scalar fields.

The same behaviour is observed for emission on the brane (Table 2) although here the suppression is larger: for $n=2$ the total emission for a brane scalar field with mass $m_{\Phi}=0.8$ drops to $39 \%$ of the emission for a massless field, if $a_{*}=0.4$, but to $72 \%$ if $a_{*}=1.5$; for $n=7$, the emission for a $m_{\Phi}=0.8$ scalar field on the brane drops to the $80 \%$ of the massless value if $a_{*}=0.4$ and to $91 \%$ if $a_{*}=1.5$.

Since the suppression of the total emissivities between brane and bulk emission differ, it is imperative to calculate the relative emissivities to find out whether the mass of the emitted field changes the energy balance in the bulk-brane channels. These, derived 
Table 1: Total energy emissivities for massive scalar fields in the bulk

\begin{tabular}{ll|ccc}
\hline & & $a_{*}=0.4$ & $a_{*}=1.0$ & $a_{*}=1.5$ \\
\hline$n=2$ & $m_{\Phi}=0$ & 1.00 & 1.54 & 3.46 \\
& $m_{\Phi}=0.4$ & 0.84 & 1.34 & 3.05 \\
& $m_{\Phi}=0.8$ & 0.52 & 0.95 & 2.46 \\
\hline$n=4$ & $m_{\Phi}=0$ & 6.29 & 9.57 & 19.22 \\
& $m_{\Phi}=0.4$ & 5.97 & 9.13 & 18.61 \\
& $m_{\Phi}=0.8$ & 5.12 & 7.99 & 16.74 \\
\hline$n=7$ & $m_{\Phi}=0$ & 131.47 & 202.48 & 327.37 \\
& $m_{\Phi}=0.4$ & 128.56 & 197.27 & 322.87 \\
& $m_{\Phi}=0.8$ & 121.57 & 188.58 & 310.18 \\
\hline
\end{tabular}

Table 2: Total energy emissivities for massive scalar fields on the brane

\begin{tabular}{ll|ccc}
\hline & & $a_{*}=0.4$ & $a_{*}=1.0$ & $a_{*}=1.5$ \\
\hline$n=2$ & $m_{\Phi}=0$ & 1.00 & 3.37 & 13.18 \\
& $m_{\Phi}=0.4$ & 0.75 & 3.10 & 11.98 \\
& $m_{\Phi}=0.8$ & 0.39 & 2.16 & 9.51 \\
\hline$n=4$ & $m_{\Phi}=0$ & 6.56 & 25.73 & 89.89 \\
& $m_{\Phi}=0.4$ & 5.73 & 23.75 & 84.18 \\
& $m_{\Phi}=0.8$ & 4.14 & 19.51 & 83.39 \\
\hline$n=7$ & $m_{\Phi}=0$ & 36.75 & 144.53 & 483.83 \\
& $m_{\Phi}=0.4$ & 34.48 & 138.86 & 471.08 \\
& $m_{\Phi}=0.8$ & 29.28 & 126.53 & 440.77 \\
\hline
\end{tabular}

by dividing the actual values of the bulk and brane emissivities for each set of values $\left(n, a_{*}, m_{\Phi}\right)$, are displayed in Table 3. Our results confirm and extend the ones of [30] where the emission of massless scalar fields was studied. In there, it was found that the bulk emission channel was becoming increasingly sub-dominant as the value of the rotation parameter increased from $a_{*}=0$ to $a_{*}=1$ - here, we show that this behaviour persists for higher values of the angular momentum parameter. Also, we confirm that the bulk-over-brane ratio take its lower value for an intermediate value of the number of extra dimensions (a result that was found in the case of both rotating [30] and non-rotating [8] black holes) but starts increasing again as $n>4$.

Overall, it is clear that the brane channel remains the dominant one over the bulk channel, during the emission of both massless and massive fields. Nevertheless, we find that the presence of the mass gives a considerable boost to the bulk-over-brane energy ratio, especially for low values of the angular momentum parameter. The boost depends also on the number of extra dimensions: for $n=2$, the mass of a $m_{\Phi}=0.8$ scalar field increases the bulk-over-brane energy ratio of a black hole with $a_{*}=0.4$ by $33 \%$, while for $n=7$ the increase is $16 \%$. We thus conclude that, when the effect of the mass of the 
Table 3: Bulk-over-brane relative energy emissivities for massive scalar fields

\begin{tabular}{ll|ccc}
\hline & & $a_{*}=0.4$ & $a_{*}=1.0$ & $a_{*}=1.5$ \\
\hline$n=2$ & $m_{\Phi}=0$ & 0.180 & 0.076 & 0.0451 \\
& $m_{\Phi}=0.4$ & 0.202 & 0.078 & 0.0458 \\
& $m_{\Phi}=0.8$ & 0.24 & 0.079 & 0.0466 \\
\hline$n=4$ & $m_{\Phi}=0$ & 0.173 & 0.067 & 0.038 \\
& $m_{\Phi}=0.4$ & 0.188 & 0.069 & 0.039 \\
& $m_{\Phi}=0.8$ & 0.223 & 0.074 & 0.040 \\
\hline$n=7$ & $m_{\Phi}=0$ & 0.645 & 0.253 & 0.122 \\
& $m_{\Phi}=0.4$ & 0.673 & 0.256 & 0.124 \\
& $m_{\Phi}=0.8$ & 0.749 & 0.269 & 0.127 \\
\hline
\end{tabular}

emitted field is taken into account, it is the fast-rotating black holes living in a spacetime with a fairly large number of extra dimensions that lose the smallest part of their energy into invisible bulk emission.

Let us finally note that the results presented in this work not only extend previous analyses for massless fields, but also improve those, too. For instance, our results for the total bulk emissivities when $m_{\Phi}=0$ agree in the first or second decimal point (depending on the value of $n$ and $a_{*}$ ) with those derived in [30] - the agreement is reassuring as a different numerical code was used. Small deviations between our results may be due to the fact that, in the calculation of the total emissivities, we have not imposed any cut-off on the frequency but instead tried to obtain the complete spectrum by keeping a realistically large number of scalar modes.

\section{Conclusions}

In this work, we have moved towards the direction of considering the emission of realistic particle states by a higher-dimensional, simply rotating black hole. We have studied the emission of massive scalar fields both in the bulk and on the brane, and investigated the role that the mass of the field plays in the corresponding energy spectra profiles and in the bulk-over-brane energy ratio.

The emission of Hawking radiation in the bulk in the form of massive scalar fields was studied first. The radial part of the field equation was first solved analytically, and an expression for the absorption probability was found that helped us investigate lowenergy aspects of the emission. Next, by using numerical analysis, the exact value of the absorption probability was determined and its dependence on the mass of the emitted field, in conjunction with the number of extra dimensions and angular-momentum of the black hole, was studied. As expected, the presence of the mass term caused the suppression of the absorption probability as additional energy is required for the emission of a massive field. Our numerical and analytical results were directly compared, and found to be in excellent agreement in the low and intermediate energy regimes for scalar fields 
with a mass smaller than (0.5-1) TeV.

The exact numerical value of the absorption probability was subsequently used to derive the differential emission rate per unit time and unit frequency in the bulk. Particular care was taken so that a large enough number of scalar modes $\left(N_{b u} \simeq 5500\right)$ was summed up in our computation of the energy spectra. The mass term caused the suppression of the energy spectra in the low and intermediate-energy regimes, compared to the massless case: for low values of $n$ and $a_{*}$ and $m_{\Phi}=0.8$, the suppression is of the order of $50 \%$, while it becomes smaller in magnitude as either $n$ or $a_{*}$ increases.

The same task was performed for the emission of massive scalar fields on the brane. The value of the absorption probability was again found both analytically and numerically, and it was shown that the two sets of results are in very good agreement, in the lowest part of the spectrum, up to masses of order (250-500) GeV. The exact profile of the energy spectra on the brane was found next in terms of the parameters $\left(m_{\Phi}, n, a_{*}\right)$, with the mass term causing again a significant suppression in their value. The suppression was larger than the one in the bulk decreasing the value of the energy emission rate to approximately $40 \%$ of that in the massless case, for low values of $n$ and $a_{*}$ and for $m_{\Phi}=0.8$. As in the case of bulk emission, a considerable number of modes $\left(N_{b r} \simeq 1700\right)$ was summed up in our calculation so that the computed spectra are as close as possible to the real ones.

The role of the mass of the emitted field in the bulk-over-brane energy ratio was also investigated. The total energy emissivities of bulk and brane emission were derived and directly compared. In agreement with previous analyses [44, 8, 30] - that we have generalised by considering a larger range of parameters of both $n$ and $a_{*}$ - we found that the bulk channel remains sub-dominant to the brane one; nevertheless, the bulk-overbrane ratio takes a considerable value especially for a large number of extra dimensions and a slowly rotating black hole. We further found that the presence of the mass of the emitted field increases the percentage of energy which is spent by the black hole in the bulk. For a small number of extra dimensions and a low value of the angular-momentum of the black hole, the enhancement of the bulk channel over the brane one can reach the value of $33 \%$ if $m_{\Phi}=0.8$.

In conclusion, in this work we have performed a comprehensive study of the emission of massive scalar fields by a higher-dimensional, simply rotating black hole both in the bulk and on the brane. We have studied the dependence of the absorption probabilities and energy emission rates on all parameters of the theory, and compared analytic and numerical methods for the computation of their value. We have confirmed the importance of the emission of a higher-dimensional black hole both in the bulk and on the brane, and demostrated that properties of the emitted field, such as its mass which was up to now largely ignored, can play a significant role in the bulk-over-brane energy balance.

Acknowledgments. We are deeply grateful to Athanasios Dedes for useful discussions regarding our numerical code. We also acknowledge participation in the RTN Universenet (MRTN-CT-2006035863-1 and MRTN-CT-2004-503369). 


\section{References}

[1] N. Arkani-Hamed, S. Dimopoulos and G. R. Dvali, Phys. Lett. B 429, 263 (1998); Phys. Rev. D 59, 086004 (1999); I. Antoniadis, N. Arkani-Hamed, S. Dimopoulos and G. R. Dvali, Phys. Lett. B 436, 257 (1998).

[2] L. Randall and R. Sundrum, Phys. Rev. Lett. 83 (1999) 3370; Phys. Rev. Lett. 83 (1999) 4690.

[3] T. Banks and W. Fischler, hep-th/9906038;

S. B. Giddings and S. Thomas, Phys. Rev. D 65, 056010 (2002);

S. Dimopoulos and G. Landsberg, Phys. Rev. D 87, 161602 (2001);

P. C. Argyres, S. Dimopoulos and J. March-Russell, Phys. Lett. B 441, 96 (1998);

D. M. Eardley and S. B. Giddings, Phys. Rev. D 66, 044011 (2002);

H. Yoshino and Y. Nambu, Phys. Rev. D66, 065004 (2002); ibid. D 67, 024009 (2003);

E. Kohlprath and G. Veneziano, JHEP 0206, 057 (2002);

V. Cardoso, O. J. C. Dias and J. P. S. Lemos, Phys. Rev. D 67, 064026 (2003);

E. Berti, M. Cavaglia and L. Gualtieri, Phys. Rev. D 69, 124011 (2004);

S. B. Giddings and V. S. Rychkov, Phys. Rev. D 70, 104026 (2004);

H. Yoshino and V. S. Rychkov, Phys. Rev. D 71 (2005) 104028 ;

D. C. Dai, G. D. Starkman and D. Stojkovic, Phys. Rev. 73 (2006) 104037 ;

H. Yoshino and R. B. Mann, Phys. Rev. 74 (2006) 044003 ;

H. Yoshino, T. Shiromizu and M. Shibata, Phys. Rev. D 74, 124022 (2006);

U. Sperhake, V. Cardoso, F. Pretorius, E. Berti, T. Hinderer and N. Yunes, Phys. Rev. Lett. 103 (2009) 131102 arXiv:0907.1252 [gr-qc]].

[4] S. W. Hawking, Commun. Math. Phys. 43, 199 (1975).

[5] P. Kanti, Int. J. Mod. Phys. A 19, 4899 (2004); Lect. Notes Phys. 769 (2009) 387 arXiv:0802.2218 [hep-th]].

[6] M. Cavaglià, Int. J. Mod. Phys. A 18, 1843 (2003); G. Landsberg, Eur. Phys. J. C 33, S927 (2004); C. M. Harris, hep-ph/0502005; A. Casanova and E. Spallucci, Class. Quant. Grav. 23, R45 (2006) |hep-ph/0512063];

E. Winstanley, 0708.2656 [hep-th].

[7] P. Kanti and J. March-Russell, Phys. Rev. D 66, 024023 (2002); Phys. Rev. D 67, 104019 (2003).

[8] C. M. Harris and P. Kanti, JHEP 0310 (2003) 014.

[9] A. Barrau, J. Grain and S. O. Alexeyev, Phys. Lett. B 584, 114 (2004); J. Grain, A. Barrau and P. Kanti, Phys. Rev. D 72, 104016 (2005); T. G. Rizzo, Class. Quant. Grav. 23, 4263 (2006).

[10] E. l. Jung, S. H. Kim and D. K. Park, Phys. Lett. B 586 (2004) 390; JHEP 0409 (2004) 005; Phys. Lett. B 602 (2004) 105; Phys. Lett. B 614 (2005) 78; E. Jung and D. K. Park, Nucl. Phys. B 717 (2005) 272; hep-th/0506204. 
[11] P. Kanti, J. Grain and A. Barrau, Phys. Rev. D 71 (2005) 104002.

[12] A. S. Cornell, W. Naylor and M. Sasaki, JHEP 0602 (2006) 012.

[13] D. K. Park, Class. Quant. Grav. 23 (2006) 4101.

[14] V. Cardoso, M. Cavaglia and L. Gualtieri, Phys. Rev. Lett. 96, 071301 (2006) [Erratum-ibid. 96, 219902 (2006)]; JHEP 0602, 021 (2006).

[15] S. Creek, O. Efthimiou, P. Kanti and K. Tamvakis, Phys. Lett. B 635 (2006) 39; O. Efthimiou, hep-th/0609144.

[16] D. C. Dai, N. Kaloper, G. D. Starkman and D. Stojkovic, Phys. Rev. D 75, 024043 (2007).

[17] V. P. Frolov and D. Stojkovic, Phys. Rev. D 67, 084004 (2003).

[18] D. Ida, K. y. Oda and S. C. Park, Phys. Rev. D 67, 064025 (2003) [Erratum-ibid. D 69, 049901 (2004)].

[19] H. Nomura, S. Yoshida, M. Tanabe and K. i. Maeda, Prog. Theor. Phys. 114, 707 (2005).

[20] C. M. Harris and P. Kanti, Phys. Lett. B 633, 106 (2006).

[21] D. Ida, K. y. Oda and S. C. Park, Phys. Rev. D 71, 124039 (2005); Phys. Rev. D 73, 124022 (2006).

[22] E. Jung, S. Kim and D. K. Park, Phys. Lett. B 615, 273 (2005); Phys. Lett. B 619, 347 (2005);

[23] E. Jung and D. K. Park, Nucl. Phys. B 731, 171 (2005).

[24] G. Duffy, C. Harris, P. Kanti and E. Winstanley, JHEP 0509, 049 (2005).

[25] M. Casals, P. Kanti and E. Winstanley, JHEP 0602, 051 (2006).

[26] M. Casals, S. R. Dolan, P. Kanti and E. Winstanley, JHEP 0703, 019 (2007).

[27] S. Creek, O. Efthimiou, P. Kanti and K. Tamvakis, Phys. Rev. D 75 (2007) 084043.

[28] S. Creek, O. Efthimiou, P. Kanti and K. Tamvakis, Phys. Rev. D 76 (2007) 104013 arXiv:0707.1768 [hep-th]].

[29] S. Creek, O. Efthimiou, P. Kanti and K. Tamvakis, Phys. Lett. B 656 (2007) 102 arXiv:0709.0241 [hep-th]].

[30] M. Casals, S. R. Dolan, P. Kanti and E. Winstanley, JHEP 0806 (2008) 071 arXiv:0801.4910 [hep-th]].

[31] P. Kanti, H. Kodama, R. A. Konoplya, N. Pappas and A. Zhidenko, Phys. Rev. D 80 (2009) 084016 [arXiv:0906.3845 [hep-th]]. 
[32] M. Casals, S. R. Dolan, P. Kanti and E. Winstanley, Phys. Lett. B 680 (2009) 365 arXiv:0907.1511 [hep-th]].

[33] D. N. Page, Phys. Rev. D 16 (1977) 2402.

[34] M. O. P. Sampaio, JHEP 0910 (2009) 008 [arXiv:0907.5107 [hep-th]]; JHEP 1002 (2010) 042 [arXiv:0911.0688 [hep-th]].

[35] R. C. Myers and M. J. Perry, Annals Phys. 172, 304 (1986).

[36] V. P. Frolov and D. Stojkovic, Phys. Rev. D 68 (2003) 064011 arXiv:gr-qc/0301016.

[37] C. Muller, in Lecture Notes in Mathematics: Spherical Harmonics (Springer-Verlag, Berlin-Heidelberg, 1966).

[38] D. Ida, Y. Uchida and Y. Morisawa, Phys. Rev. D 67 (2003) 084019.

[39] E. Berti, V. Cardoso and M. Casals, Phys. Rev. D 73 (2006) 024013 [Erratum-ibid. D 73 (2006) 109902];

[40] M. Abramowitz and I. Stegun, Handbook of Mathematical Functions (Academic, New York, 1996).

[41] S. S. Gubser, I. R. Klebanov and A. A. Tseytlin, Nucl. Phys. B499, 217 (1997);

S. D. Mathur, Nucl. Phys. B 514, 204 (1998);

S. S. Gubser, Phys. Rev. D 56, 4984 (1997).

[42] Y.B. Zel'dovich, JETP Lett. 14, 180 (1971).

[43] A. A. Starobinskii and S. M. Churilov, Sov. Phys.-JETP 38, 1 (1974);

E. D. Fackerell and R. G. Crossman, J. Math. Phys. 18, 1849 (1977);

E. Seidel, Class. Quant. Grav. 6, 1057 (1989).

[44] R. Emparan, G. T. Horowitz and R. C. Myers Phys. Rev. Lett. 85, 499 (2000) hep-th/0003118. 